\title{
A STUDY OF THE XENON VAPOUR PRESSURE ISOTOPE EFFECT
}

\author{
by
}

Amal Abdullah Alamre

A thesis submitted to the Faculty of Graduate and Postdoctoral Affairs in partial fulfillment of the requirements for the degree of

Master of Science

in

Physics

Specialization in Particle Physics

Ottawa-Carleton Institute for Physics

Department of Physics

Carleton University

Ottawa, ON, Canada

(C) 2019 Amal Abdullah Alamre 


\begin{abstract}
The goal of this research was to study the xenon vapour pressure isotope effect (VPIE) and this was done to explore the feasibility of doing the Xe enrichment, using a $1.83 \mathrm{~m}$ high, cryogenic distillation column at Carleton University. One of the famous isotopes for the $0 \mathrm{v} \beta \beta$ decay detection is ${ }^{136} \mathrm{Xe}$ which is not easily available in pure form, and it is available from the Centrifuge systems in Russia. Therefore, the VPIE of xenon isotopes were needed which have not been measured to date. The VPIE from this work would be $5.28 \times 10^{-5}$ while the theoretical value reported by Tew is $5.17 \times 10^{-5}$ per mass unit. Thus, the experimental and theoretical values agree with the accuracy of the present slope measurement. Moreover, the data demonstrate for the first time the linear dependence of the VPIE across a range of isotopes, and this is consistent with the theoretical predictions.
\end{abstract}




\section{Acknowledgements}

I would like to thank all the people that contributed to the achievement of this work. I would like to express sincere gratitude and appreciation to my supervisor Dr. David Sinclair, who has been patient and supportive throughout my research, it would not have been possible and successful without him. Also, I would like to thank him for giving me an open time to meet and discuss updates for conducting this research. Special thanks to my Co-Supervisor Adjunct Dr. Ibtesam Badhrees for her support and advice throughout this journey. Also, I would like to thank Caio Licciardi and Brandon Death For their participation in this work. Moreover, I would like to express sincere gratitude and appreciation to my mother. Although she is not with me physically here, her love and blessings are always with me, which encouraged me to do better. Furthermore, I would like to thank my husband because he is my greatest support and my strongest motivation to complete my study. Finally, big thanks to my father, my children, and the rest of the family and my friends for their emotional support, unending encouragement, for believing in me, and love throughout this journey. 


\section{Table of Contents}

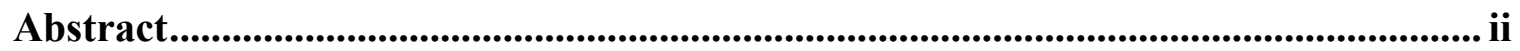

Acknowledgements ............................................................................................................................. iii

Table of Contents ........................................................................................................ iv

List of Tables ............................................................................................................................... vii

List of Figures........................................................................................................................... ix

Chapter 1: Introduction ............................................................................................................... 1

Chapter 2: Neutrinos ..................................................................................................................... 7

$2.1 \quad$ Historical Background ...................................................................................

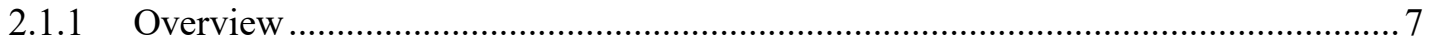

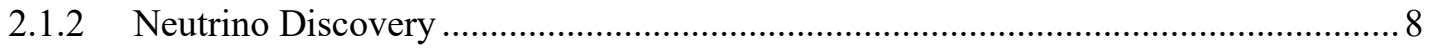

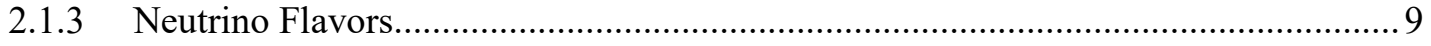

2.2 Neutrino Oscillations ........................................................................................ 10

2.2.1 Solar neutrino problem................................................................................. 11

2.2.2 Theory of neutrino oscillation ........................................................................... 13

2.2.3 Two Neutrino Mixing …………………………………........................................ 14

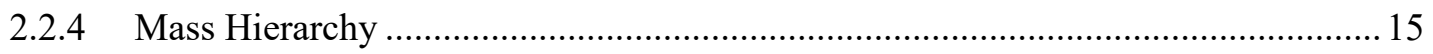

2.3 Massive Neutrinos in the Standard Model ……………………………………..... 16

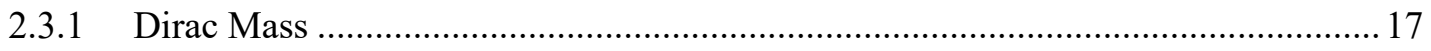

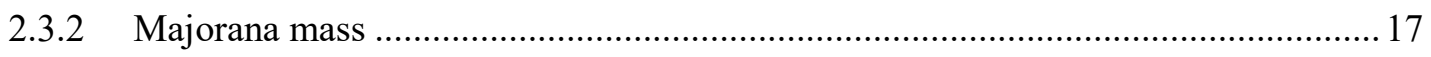

2.3.3 Absolute Neutrino Mass......................................................................................... 18

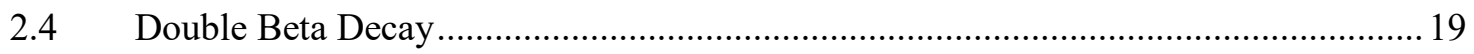

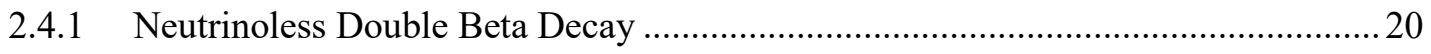

2.4.2 Decay Rates............................................................................................... 21 


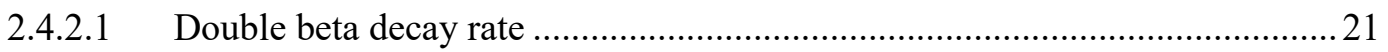

2.4.2.2 Neutrinoless Double Beta Decay Rate ....................................................... 22

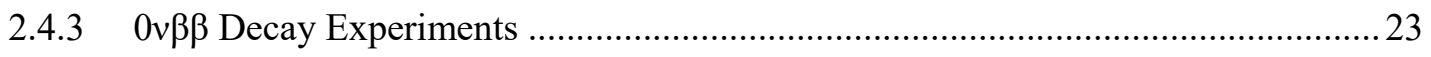

2.4.3.1 ${ }^{136} \mathrm{Xe}$ as Target Material in $0 v \beta \beta$ Decay Experiments .....................................26

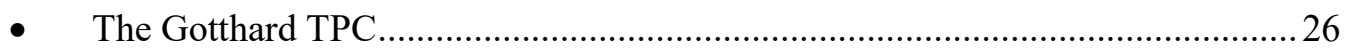

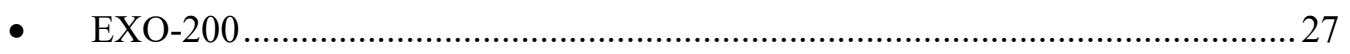

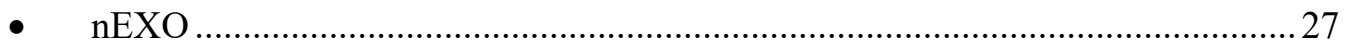

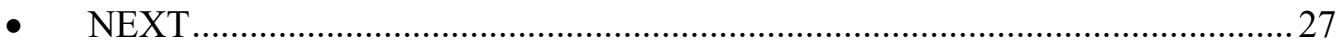

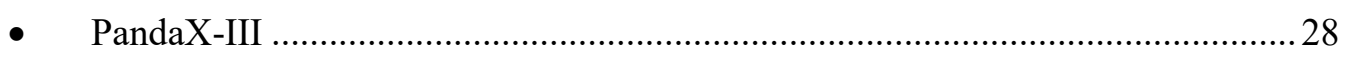

- KamLAND-Zen and KamLAND2-Zen .......................................................28

Chapter 3: Operation of a still ............................................................................... 30

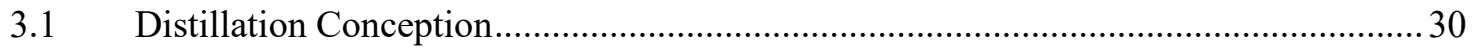

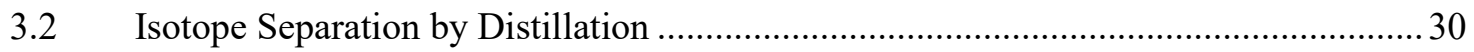

3.3 Need for VPIE pressure differences for different isotopes....................................... 32

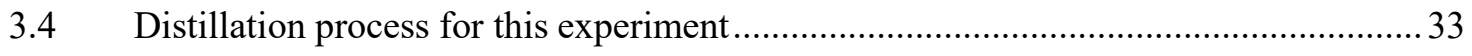

3.4.1 Height Equivalent Theoretical Plate (HETP) ..................................................... 34

Chapter 4: Description of the Carleton Experiment .......................................................... 37

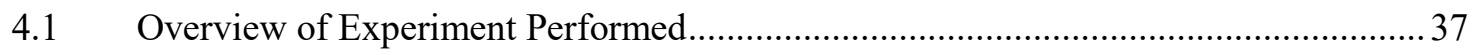

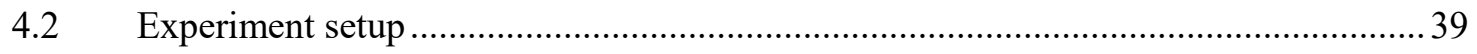

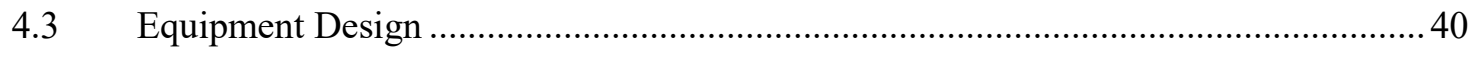

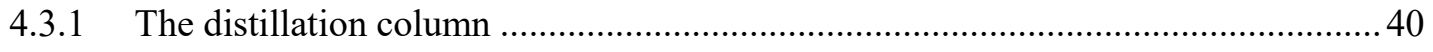

4.3.2 Stainless Steel Boiler (Made custom at Carleton University) ................................. 41

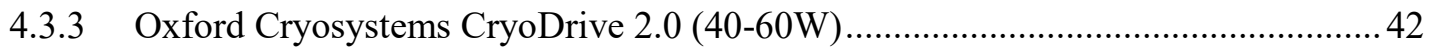

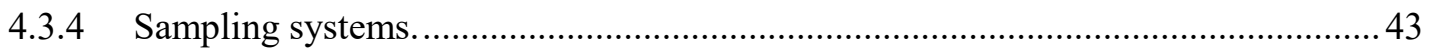

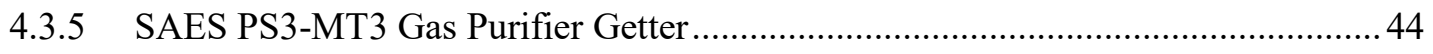




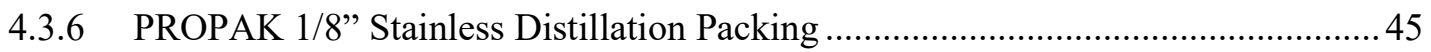

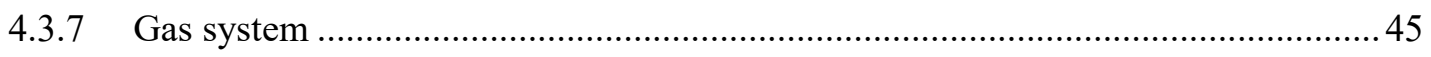

4.3.8 Pressure Sensor and Temperature Sensor ........................................................ 46

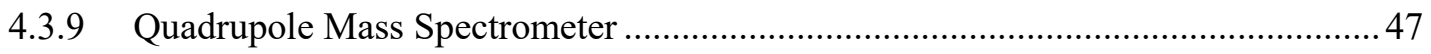

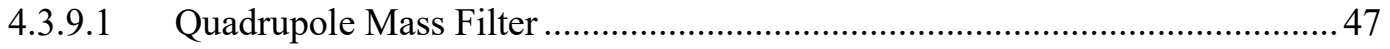

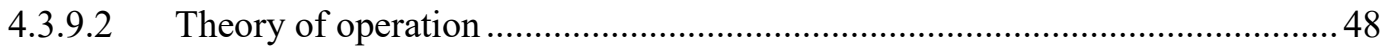

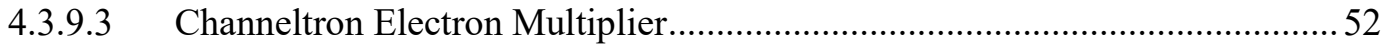

4.3.10 Merlin Automation Data System Software ...................................................... 54

Chapter 5: Experimental Procedure ................................................................... 55

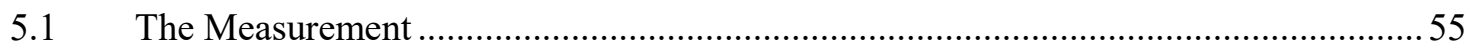

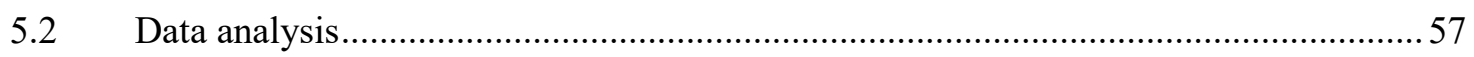

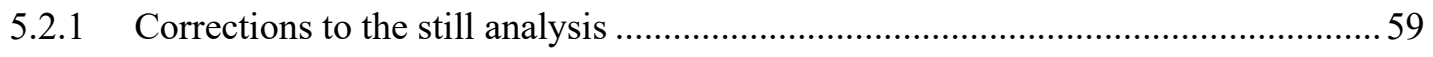

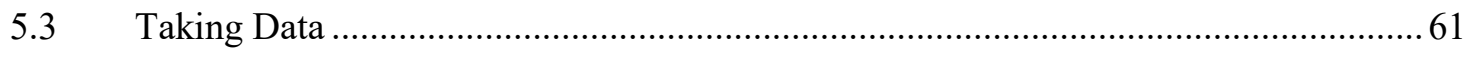

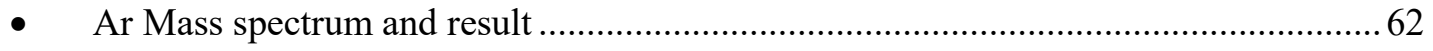

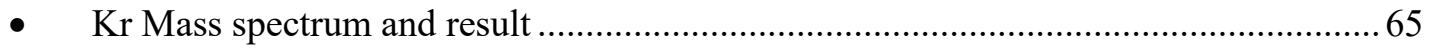

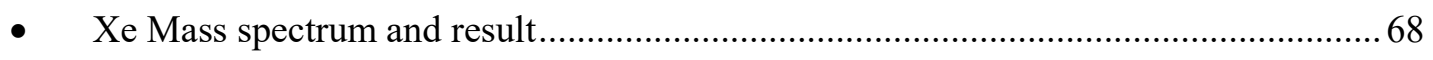

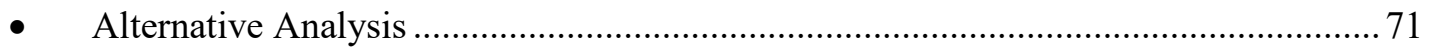

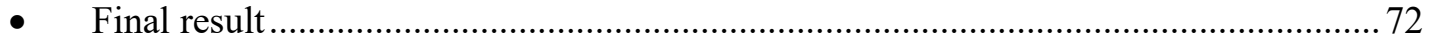

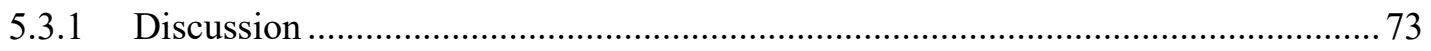

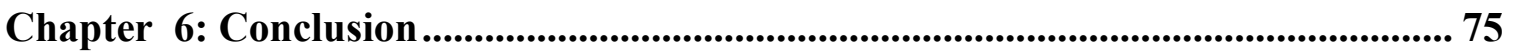

Bibliography .............................................................................................................................. 77 


\section{List of Tables}

Table 1.1: Distillation parameters for the different noble gases, and the values for VPIE for $\mathrm{Ar}$ and $\mathrm{Kr}$ are experimental while VPIE for xenon is theoretical. Calculated values refer to fluids at their triple points, and the technique is differential pressure [1]. No errors are reported for either the experimental or theoretical results. For both argon and krypton vapour pressures, results were quoted over an extensive set of temperatures. For argon the VPIE's fall monotonically with temperature and the fluctuations from one measurement to another look to be less than 1\%. For krypton the fluctuations are of order $15 \%$ and the error cannot be less than this

Table 2.1: Measurements of the neutrino mass-squared and mixing angles from neutrino oscillation experiments, uncertainties are to $3 \sigma[41]$. 14

Table 2.2: The most updated results of lower limits on $\mathrm{T}_{1 / 2}$ and their corresponding upper limits on the effective neutrino mass $\mathrm{m} \beta \beta[51]$ 25

Table 5.1: Data taking runs. 61

Table 5.2: Ar calculating the enrichment between top and bottom measurements, and calculating the slope for every enrichment measurement. Runs from the first day. Oct 5 show results prior to the column reaching equilibrium. Analysis of the equilibrium enrichment start with the Oct 6 data.

Table 5.3: Kr calculating the enrichment between top and bottom measurements and calculating the slope for every enrichment measurement. 66

Table 5.4: Xe calculating the enrichment between top and bottom measurements and calculating the slope for every enrichment measurement. 
Table 5.5: For this analysis the ${ }^{132}$ Xe peak has been used to normalize the runs. Peak 124 and 126 have been removed from the analysis because the fluctuation in these small peaks is large. These runs of Xe were used to do the analysis because there was an improvement in setting for mass spectrum which gave better resolution....................... 69 Table 5.6: Measured enrichment factors for the cryogenic still together with the inferred VPIE at the triple point. Tew reports experimental data for $\mathrm{Ar}$ and $\mathrm{Kr}$ and theoretical value for Xe. Uncertainties for the VPIE measurements include only the error on the slopes obtained from the fit results. See text for further discussion. 


\section{List of Figures}

Figure 2.1: Scheme of the normal hierarchy, inverted hierarchy and quasi-degenerate hierarchy, and the color code indicates the flavour $(e, \mu, \tau)$ present in the mass eigenstates $(v 1, v 2, v 3)$, The mass of the lightest neutrino is unknown [42] 16

Figure 2.2: Feynman graphs for Double Beta Decay $(2 v \beta \beta)$ (left) and Neutrinoless Double Beta Decay $(0 v \beta \beta)$ (right) [49]. 21

Figure 2.3 Effective Majorana mass as a function of the lightest neutrino mass in the normal and inverted hierarchies. IS denotes the inverted-hierarchy and NS the normalhierarchy. Modified from [47].

Figure 2.4: The background index as a function of energy resolution for some selected past, current and future projects with ${ }^{76} \mathrm{Ge},{ }^{100} \mathrm{Mo},{ }^{130} \mathrm{Te}$ and ${ }^{136} \mathrm{Xe}$ as target materials

Figure 3.1: Basic diagram of distillation process used in the experiment [79]

Figure 4.1: Distillation system at Carleton is $2 \mathrm{~m}$ long. The condenser (operating dawn 200C) is located in the top 8 " cross while the reboiler is in the bottom cross. The packed column fills the space between, inside a vacuum jacket. This has been run with argon, krypton, and xenon gasses. 38

Figure 4.2: Experimental setup showing gas entering the top of the still, with samples extracted from the top \& bottom to be sent to the mass spectrum [81].

Figure 4.3: Assembly of the inner and outer pipes showing the spacers. Super insulation

on the inner pipe starts just above the region shown.

Figure 4.4: The Boiler. 
Figure 4.5: Condenser region. The copper cold finger is shown at the top entering a 2.75" cube which forms the condenser. The packed bed is located below the lower bellows. Gas connections to the cube enter through the bellows at the right. A Pt thermometer is

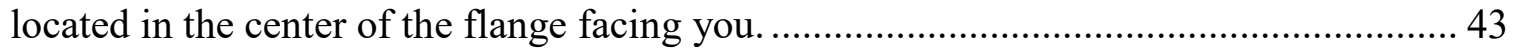

Figure 4.6: Gas sampling control panel installed at lower level attached to tower. ........ 44 Figure 4.7: Propak 1/8 which gives a very high surface area bent into a U shape [82]... 45 Figure 4.8: Xenon recovery system installed at lower level and Emergency xenon recovery balloons installed at lower level............................................................. 46

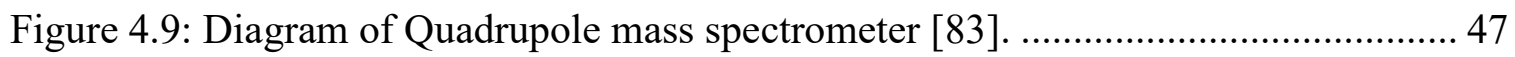
Figure 4.10: Diagram of Quadrupole mass filter. The ions enter and travel in the z-

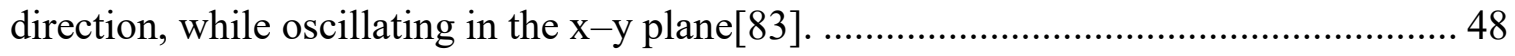

Figure 4.11: Stability diagram for a quadrupole mass filter. ..................................... 52 Figure 4.12: Diagram of the multiplier-dynode configuration for positive ion counting. It is adapted from [47] 53

Figure 5.1: Measured spectrum for Ar. The mass peaks from 36 to 40 are clearly seen.. 62 Figure 5.2: Measured argon enrichment percentage as a function of mass. The slope is. 64 Figure 5.3: Mass spectrum for krypton. The mass peaks from 78 to 86 are clearly seen. 65 Figure 5.4: Measured krypton enrichment percentage as a function of mass. The slope is $0.94 \%$ /Mass Unit. For this analysis the ${ }^{84} \mathrm{Kr}$ peak has been used to normalize the runs. 67 Figure 5.5: Mass spectrum for xenon. The mass peaks from 124 to 136 are clearly seen.

Figure 5.6: Measured xenon enrichment percentage as a function of mass. The slope is 70 
Figure 5.7: Alternative analysis for fractional difference between the top and bottom of the column for each isotope (by Caio Licciardi). 


\section{Chapter 1: Introduction}

In the field of sub-atomic physics, one of the crucial problems is to search for the evidence of neutrinoless double beta decay. The next generation searches for neutrinoless double beta decay require large quantities of target material to improve the sensitivity of the detectors looking for such rare process. One of the most promising targets is an isotope of xenon, ${ }^{136} \mathrm{Xe}$. To reach the normal hierarchy for neutrino masses $\sim 50$ tonnes of this isotope are required. In general, economic or background issues dictate use of isotopic enrichment. Currently, the only enrichment technique available is centrifuge and this is only possible in Russia. This thesis presents a study of the first step in exploring the feasibility of economical production of this isotope by distillation, namely a study of the xenon vapour pressure isotope effect. Separation by distillation depends on the constituents having different vapour pressures. For xenon, the differences in vapour pressures of the isotopes are expected to be extremely small and direct measurements are very difficult. Therefore, the indirect method of using distillation to measure the effect was adopted in this thesis.

The isotopic dependence of vapour pressure of Xenon has not been studied a lot, although there are a significant publication record and data available for other noble elements. Indeed, for Xenon, earlier studies gave much smaller results than the theoretical value $[1,2]$. In [3] where the measurement was based on distillation, they obtained a value of vapour pressure with zero dependencies. A review of the available data for this study is given by Tew [1].

The possibility of Xenon triple point measurements motivates the interest in the Xenon system, which can also be used as a temperature standard. The system would not be 
useful as a standard if the triple point temperature of Xenon differed significantly for different isotopes. A recent paper [4], which made a precise measurement of the triple point temperature of Xenon has suggested no evidence of an isotopic effect.

The enriched isotopes of selected materials are often involved in the search for fundamental new physics. The recent examples are as follows: the SNO experiment which used 1000 tonnes of heavy water to discover the oscillation of solar neutrinos leading to the 2015 Nobel prize; use of ${ }^{136} \mathrm{Xe}$ by the EXO-200 experiment which used $200 \mathrm{~kg}$ of enriched Xenon to discover two-neutrino double beta decay; and the use of ${ }^{40} \mathrm{Ar}$ which is depleted in radioactivity from ${ }^{39} \mathrm{Ar}$ for the search for dark matter.

One of the most pressing problems in today's particle physics is the search for neutrinoless double beta decay. If it exists, the signal for this decay will allow establishing the absolute mass of neutrinos and would solve the problem of whether neutrinos are Majorana particles or not. With the discovery of this decay, the question of the origin of neutrino mass can be helped out, and hence help to explain the dominance of matter over antimatter in the universe.

${ }^{136} \mathrm{Xe}$ is considered as very important and attractive target for neutrinoless double beta decay, since it can be made mostly free of the radioactive contamination, has excellent self-shielding from external backgrounds, and can be made into detectors of high sensitivity. Many groups [5-8] are in the process of constructing or proposing Xenon based detectors, and the cost of those proposals is dominated by the high cost of Xenon and the requirement of isotopic enrichment to limit the total Xenon to be used. The current projects for Xenon based experiments are aimed at a sensitivity to probe the predicted lifetime if neutrinos have an inverted hierarchy. 
Recently, however, three different groups $[9,10]$ and $[11]$ have shown results in favour of normal hierarchy. It is essential to calculate the sensitivity required to reach the normal hierarchy developed by these detectors. In the case of Xenon, the required lifetime sensitivity is about $10^{30}$ years, which means it requires a Xenon exposure of order one kilotonne year, and such detectors might use 50 tonnes of Xenon isotope with a 20 -year exposure to reach the required sensitivity.

Those detectors will be costly unless more economic ways to enriching the Xenon can be found. At the present time, centrifuge separation is the only method used for this, and only one source is available. The alternate method for large scale Xenon isotope enrichment can be distillation, but the absence of credible data on the isotopic variation of vapour pressure in Xenon limits the meaningful feasibility study of this alternative.

The isotopic modification of the thermodynamic properties of Xenon, such as vapour pressure, could be understood by using statistical theory to the condensed phase [12]. Lopes [13] extended this work and had showed that in case of noble systems, the vapour pressure isotope effect (VPIE) can be related to parameters of Lennard-Jones potential and this model agreed with experimental data over many orders of magnitude with Xenon as an exception. The rough scale of VPIE from the model is:

$$
V P I E \propto \frac{\Delta m}{m^{3}}
$$

Where $\Delta \mathrm{m}$ is the isotopic mass range and $\mathrm{m}$ is the mean mass. The Lenard-Jones potential between the atoms can correct for these parameters. It explains why distillation is a standard technique for separation of isotopic light elements and molecules. For example, the SNO experiment's heavy water was enriched from $35 \%$ to $99.92 \%$ using distillation, but this process is difficult for more massive systems. Recently, it has been demonstrated 
by ARIA in the DarkSide Collaboration [14] that the radioactive isotope ${ }^{39} \mathrm{Ar}$ can be depleted from natural argon using the distillation process. The facility required, ARIA, is made from a very tall $(350 \mathrm{~m})$ reflux column for the distillation, and this can be done using a mine shaft for support. Xenon has VPIE per unit mass unit smaller by a factor of $\sim 35$ compared with argon from the $\mathrm{m}^{3}$ term in equation (1.1). The bulk of the Xenon has mass five units away from the desired isotope ${ }^{136} \mathrm{Xe}$, so the effective VPIE for this isotope is about seven times smaller than argon. Further, in the xenon neutrinoless double beta decay experiments, the advantage of enrichment material is mostly an economic one, so high enrichment is not required. Therefore, the ARIA success gives some support to the idea of enrichment of Xenon through distillation.

\begin{tabular}{|c|c|c|c|c|c|}
\hline Fluid & Mass Range & $\begin{array}{c}\text { Pressure } \\
(\mathrm{kPa})\end{array}$ & Temp.(K) & VPIE & $\begin{array}{c}\text { VPIE/(TP) } \\
\text { per AMU }\end{array}$ \\
\hline Argon & $36-40$ & 68.9 & 83.806 & 0.0066 & 0.00164 \\
\hline Kryp- & $80-84$ & 73.15 & 115.78 & 0.0010 & 0.00025 \\
\hline Xenon & $130-136$ & 81.66 & 161.4 & 0.0003 & 0.000050 \\
\hline
\end{tabular}

Table 1.1: Distillation parameters for the different noble gases, and the values for VPIE for $\mathrm{Ar}$ and $\mathrm{Kr}$ are experimental while VPIE for xenon is theoretical. Calculated values refer to fluids at their triple points, and the technique is differential pressure [1]. No errors are reported for either the experimental or theoretical results. For both argon and krypton vapour pressures, results were quoted over an extensive set of temperatures. For argon the VPIE's fall monotonically with temperature and the fluctuations from one measurement to another look to be less than $1 \%$. For krypton the fluctuations are of order $15 \%$ and the error cannot be less than this. 
The vapour pressure differences shown in the second column for Argon \& Krypton were measured, whereas that for Xenon is estimated [1]. From the table (1.1), we can see that the vapour pressure difference for xenon is 0.0003 which is a very small number. This small number explains why it is so difficult to do this measurement, and nobody managed to do this measurement for xenon. In this study, the measurements were made for xenon isotopes separation using a $1.8 \mathrm{~m}$ high, cryogenic distillation column. The calibration of the column was done with argon and krypton since we have measurement values of VPIE for these elements. All measurements were made at a pressure of $100 \mathrm{kPa}$, and the same molar vapour flow rate was used in each case. The VPIE for Xenon from these data agrees well with the theoretical values, which is in contrast to the older measurements.

The thesis is divided into six principal chapters, and the structure of the thesis is as follows. In Chapter 2 introduces the theory and properties of the neutrino leading up to the neutrino oscillation experiments. Then, it introduces the theory of double-beta decay, and discussed the experiments searching for the $0 v \beta \beta$-decay. In Chapter 3 , the operation of still and isotope separation by distillation are presented with the theory about vapour pressure differences for noble systems is presented. Chapter 4 introduces description of the Carleton experiment setup used to measurement Xenon isotopes separation and vapour pressures. In Chapter 5, the measurements of vapour pressures and enrichment of Xenon using distillation are presented. In chapter 6 , summarized the conclusions of the first credible measure of the separation power of a still for xenon isotopes, which lead to the first VPIE data for xenon and the linear relationship between VPIE and isotope mass for argon, krypton and xenon. 
The work is done by the group. Our collaboration consists of Dr. David Sinclair, Dr. Ibtesam Badhrees, Caio Licciardi and Brandon Death. My role included operating the still for all the runs, and the analysis that I presented in this thesis, including writing the analysis software. 


\section{Chapter 2: Neutrinos}

\subsection{Historical Background}

\subsubsection{Overview}

The neutrino is a subatomic particle that is most popular to study in today's particle physics because of its mysterious nature. It has been a controversial topic since a hundred years ago and continuing until the present time. The term neutrino was first introduced after the first controversy about the 'conservation of energy crisis', which basically means the continuity of the energy spectrum of electrons emitted in the beta decay of pure substances. The electron, proton and photon were only three subatomic particles known at that time, and the continuous energy in beta decay spectrum was interpreted as serious issues with the law of conservation of energy [15]. Initially, to explain this condition, some argument was made that in the beta decay of an $\mathrm{X}$ atomic nucleus emits an electron $\mathrm{e}^{-}$and converted to the nucleus of a different element $\mathrm{Y}$ [16],

$$
X \rightarrow Y^{+}+e^{-}
$$

The energy released from this process is the mass difference $\Delta \mathrm{M}=\mathrm{M}_{\mathrm{X}}-\mathrm{M}_{\mathrm{Y}}$. Where, $M_{X}$ and $M_{Y}$ are the nuclear masses before and after the decay, respectively. To conserve the energy the electron has to be emitted with kinetic energy $K_{e}=\Delta M-m_{e}$.

Since $\Delta M$ is a constant for the parent nucleus, so $K_{e}$ should also be constant.

C. Ellis and W. Wooster published their results in 1927 [17] showing electron spectra are, in fact, continuous, and energy conservation did not apply here. Wolfgang Pauli, a theoretical physicist at the Federal Institute of Technology, proposed a less drastic conclusion and proposed the prediction of the existence of a new neutral particle which is existed in the 
nucleus and also emitted in the $\beta$ decay of elements and thus solved the conservation of energy crisis.

After Pauli, a few years later, the existence of the neutrino was theoretically predicted by Enrico Fermi, which is famous for his theory on beta decay [18]. Fermi in his classic paper specifically showed that in the beta decay actually a neutron, $n$, is conversed to a proton, $\mathrm{p}$, with the emission of an electron with an antineutrino,

$$
n \rightarrow p^{+}+e^{-}+\bar{v}
$$

The conclusion of Fermi's theory is that the shape of the $\beta$ decay spectrum, especially near the Q value which is different between mass-energy of initial and final particles in a nuclear decay and at high energy depends upon the neutrino's mass [16]. Thus, Fermi explained that either the neutrino has zero mass or very small mass as compared to the mass of an electron.

\subsubsection{Neutrino Discovery}

The second important conclusion of Fermi's theory was given by theorists Hans Bethe and Rudolf Peierls, which was the possibility of an inverse $\beta$ decay reaction. In this reaction, an antineutrino $\bar{v}$ is absorbed by a proton converting it to a neutron with the emission of a positron, $e^{+}$,

$$
\bar{v}+p^{+} \rightarrow e^{+}+n
$$

The above process allowed the first detection of the antineutrino in 1953 when $\overline{v_{e}}$ scattering events were observed at the Savannah River reactor in South Carolina [19]. 
This experiment was made of liquid scintillator source from the reactor. The gamma rays from the positron annihilations and from neutron capture on cadmium chloride were collected by the photomultiplier tubes. These two events were having a time delay of $5 \mu$ s and were having consistency between the measured and predicted cross section, which explained the existence of these antineutrinos particles and also validated the beta decay theory predicted by Fermi.

\subsubsection{Neutrino Flavors}

In the next decades, many advancements were made in neutrino physics. The idea of parity violation was discovered in 1956 in decays of neutral Kaons by Tsung-Dao Lee and ChenNing at the Berkeley National Laboratory (BNL)[20]. This parity violation in kaons was experimentally confirmed in 1957 by Chien -Siung Wu in collaboration with Lee and Yang in the beta decay of ${ }^{60} \mathrm{Co}$ nuclei, which makes this experiment most popular in those days [21]. Lee and Yang published a paper in that year which explained the possibility of parity violation in the neutrinos, and it exists only if the Masses of neutrinos are negligible [22]. After a year of this publication it was measured at BNL that the neutrinos were determined to be left-handed particles [23]. It was depicted at BNL after few years that neutrinos exists in two flavours mainly, one associated with electron called electron neutrino and the other associated with muons called muon neutrino [24]. The full development of electroweak theory gave strong evidence for the other flavour of neutrino associated with the tau lepton called tau neutrino, which was later discovered after the discovery of tau particle [25]. 
In 1989 the precision observation of Z boson lifetime at the Large Electron-Positron collider (LEP) gave the primary evidence for the existence of three neutrinos. After combining the observation of four LEP experiments (ALEPH, DELPHI, L3 and OPAL), it was concluded that the number of neutrino flavours that exist in nature is $N_{v}=2.984 \pm 0.008$ [26]. The Donut experiment finally published the observation of tau neutrinos in 2001 [27].

The parity violation and charge conjugation symmetry were violated observed in the decay of neutral kaons [28]. This call is the CP symmetry breaking effect, which is also considered as a necessary condition for matter-antimatter asymmetry in the universe, which leads to the fact that matter dominates in the universe today. The Babar and Belle $[29,30]$ experiments experimentally observed this CP violation in the B-meson decays, but the effect of this violation on matter-antimatter asymmetry is very small.

\subsection{Neutrino Oscillations}

In the early history of neutrino physics, the neutrino was considered as a very lightweight particle with left-handed chirality and three flavours as $v_{e}, v_{\mu}$ and $v_{\tau}$. Early experiments searching for electron neutrinos from the Sun found a flux much lower than expected. It was not clear if this was caused by problems in the Solar Model or if neutrinos were changing from electron flavour to the other flavours (a process called 'neutrino oscillations'). This proved very important for the physics of neutrino mass. 


\subsubsection{Solar neutrino problem}

The proton-proton mechanism (pp mechanism) in the sun, which generates most of the energy, was given by Charles Critchfield and Hans Beth in 1930. The helium is formed from hydrogen which releasing energy in the prosses. There are five paths for this process given below given below[31]:

$$
\begin{gathered}
p+p \rightarrow d+e^{+}+v_{e} \\
p+p+e^{-} \rightarrow d+v_{e} \\
{ }^{3} \mathrm{He}+p \rightarrow \alpha+e^{+}+v_{e} \\
{ }^{7} \mathrm{Be}+e^{-} \rightarrow{ }^{7} \mathrm{Li}+v_{e} \\
{ }^{8} \mathrm{~B} \rightarrow{ }^{8} \mathrm{Be}^{*}+e^{+}+v_{e}
\end{gathered}
$$

Bahcall calculated the fluxes of neutrinos produced in these reactions [32, 33]. Another physicist, Davis was working on an experiment at the same time which was aimed to detect antineutrinos from a nuclear reactor through the basic reaction $v_{e}+{ }^{37} \mathrm{Cl} \rightarrow{ }^{37} \mathrm{Ar}+e^{-}$.

For this reaction, the neutrino must have energy more than $\sim 1 \mathrm{MeV}$. The maximum energy of pp neutrinos in this process $p+p \rightarrow d+e^{+}+v_{e}$ is about $0.42 \mathrm{MeV}$, so this process does not contribute in this experiment.

In 1964, Bahcall and Davis collaborated and proposed a chlorine type experiment to detect neutrinos produced in the interior of the Sun. They made a detector at Homestake 
Gold Mine in South Dakota, which contained 38000 litres of carbon tetrachloride. They published their first results in 1968, which states the solar neutrinos upper limit was 2-3 times lower than Bahcall's prediction [34]. This discrepancy of the number of solar neutrinos predicted by theory and the recorded by experiment is called "Solar neutrino problem."

In the same year of Davis's results, another physicist Pontecorvo suggested that the solar neutrino problem can be solved by considering the oscillations of neutrinos between different flavours [35]. Just like the particle oscillation in neutral kaon system predicted by Gell-Mann and Pais in 1955 [36] which was experimentally confirmed by Leon Lederman in 1955 [37], Pontecorvo predicted that it might be possible that the large fraction of electron neutrinos from the Sun are converted to muon or tau neutrinos while coming to Earth. Since these flavours not interact at low energies, this could explain the result by Davis and Bahcall.

The experimental confirmation of neutrino oscillations was first done by the SuperKamiokande collaboration in Japan in 1998 when they detected the neutrino oscillations from cosmic ray interactions in the atmosphere. The other definitive demonstration of neutrino oscillations from the Sun was given by the Sudbury Neutrino Observatory (SNO) in Canada, and Super-Kamiokande in Japan [38]. SNO was a heavy water Cherenkov detector able to detect both the electron neutrino flux and the total neutrino flux. Super-Kamiokande is a light water detector measuring neutrino electron scattering. These experiments, together with reactor and particle accelerators measurements, confirmed the existence of neutrino oscillations and solved the solar neutrino problem. However, these experiments could not provide the absolute value of the neutrino masses. 


\subsubsection{Theory of neutrino oscillation}

The occurrence of neutrino oscillations among different types of neutrinos needs the three flavour states of neutrino, which are electron neutrino $\left(v_{e}\right)$, the muon neutrino $\left(v_{\mu}\right)$, and the tau neutrino $\left(v_{\tau}\right)$. These three flavours states are different from the mass eigenstates $\left(v_{1}, v_{2}\right.$ and $\left.v_{3}\right)$. The particle which we observe in the detector is the actual flavour eigenstates of particles and the states with mass eigenstates propagate through space with some well-defined mass. The flavour and mass eigenstates are quantum superpositions of each other. The mass eigenstates and neutrino flavours are related by the $\mathrm{V}$ unitary matrix as follows,

$$
v_{\alpha}=\sum V_{\alpha, i}^{*} v_{i}
$$

$\mathrm{V}$ is the VPMNS matrix by Pontecorvo-Maki-Nakagawa-Sakata [39].

$$
\begin{gathered}
{\left[\begin{array}{l}
v_{e} \\
v_{\mu} \\
v_{\tau}
\end{array}\right]=\left[\begin{array}{lll}
V_{e 1} & V_{e 2} & V_{e 3} \\
V_{\mu 1} & V_{\mu 2} & V_{\mu 3} \\
V_{\tau 1} & V_{\tau 2} & V_{\tau 3}
\end{array}\right]\left[\begin{array}{l}
V_{1} \\
V_{2} \\
V_{3}
\end{array}\right]} \\
V_{P M N S}=\left[\begin{array}{ccc}
c_{12} c_{13} e^{\frac{i \phi_{1}}{2}} & s_{13} e^{-i \delta} \\
-s_{12} c_{23}-c_{12} s_{23} s_{13} e^{i \delta} & \left(c_{12} c_{23}-s_{12} s_{23} s_{13} e^{i \delta}\right) e^{\frac{i \phi_{2}}{2}} & s_{23} c_{13} \\
s_{12} s_{23}-c_{12} c_{23} s_{13} e^{i \delta} & -c_{12} s_{23}-s_{12} c_{23} s_{13} e^{i \delta} & c_{23} c_{13}
\end{array}\right]
\end{gathered}
$$

where, $C_{i j}=\cos \left[\theta_{i j}\right]$ and $S_{i j}=\sin \left[\theta_{i j}\right]$. The neutrino mixing from the VPMNS ma-

trix depends upon $\theta_{23}, \theta_{13}$ and $\theta_{12}$ rotation angles with $0 \leq \theta_{i} \leq \pi / 2$ and, depending on the Dirac or Majorana nature of neutrinos, one or three CP-violating phases, $\delta, \theta_{2}$ and $\theta_{3}$ with $0 \leq \delta, \theta_{i} \leq 2 \pi$. Here, $\delta$ is the Dirac phase. The other two phases $\theta_{2}$ and $\theta_{3}$ are the Majorana phases, which are only allowed if neutrinos are Majorana particles. 


\subsubsection{Two Neutrino Mixing}

The amount of mixing between neutrino mass states is determined by the PMNS matrix, however, neutrino oscillations also depend on square of the difference of masses between the mass eigenstates $\Delta m_{21}^{2}$ and $\Delta m_{32}^{2}$ where $\Delta m_{i j}^{2}=m_{i}^{2}-m_{j}^{2}$.

The survival probability of a neutrino produced in some flavour $\alpha$ to remain in same flavour after some time can be considered as [40],

$$
P_{\alpha \alpha}=1-\sin ^{2} \theta \sin ^{2}\left(1.27 \frac{L}{E} \Delta m^{2}\right)
$$

Where, $\mathrm{L}$ is the distance travelled by the neutrino in $\mathrm{km}$, and $\mathrm{E}$ is energy of neutrino in GeV. $P_{\alpha \alpha}$ is the survival probability in the case of two neutrinos. The current experimental measurements of the neutrino mass-squared and mixing angles from neutrino oscillation experiments are shown in table (2.1).

\begin{tabular}{|c|c|c|}
\hline Mass Hierarchy & Parameter & Measured value \\
\hline & $\Delta m_{21}^{2}$ & $73.7_{-4.4}^{+5.9} \mathrm{meV}^{2}$ \\
& $\sin ^{2} \theta_{12}$ & $0.297_{-0.047}^{+0.057}$ \\
\hline \multirow{2}{*}{ Normal Hierarchy } & $\Delta m_{31}^{2}$ & $2560_{-110}^{+130} \mathrm{meV}^{2}$ \\
& $\sin ^{2} \theta_{23}$ & $0.425_{-0.044}^{+0.190}$ \\
& $\sin ^{2} \theta_{13}$ & $0.0215 \pm 0.0025$ \\
\hline \multirow{2}{*}{ Inverted Hierarchy } & $\Delta m_{23}^{2}$ & $2540 \pm 120 \mathrm{meV}^{2}$ \\
& $\sin ^{2} \theta_{23}$ & $0.589_{-0.205}^{+0.047}$ \\
& $\sin ^{2} \theta_{13}$ & $0.0216 \pm 0.0026$ \\
\hline
\end{tabular}

Table 2.1: Measurements of the neutrino mass-squared and mixing angles from neutrino oscillation experiments, uncertainties are to $3 \sigma[41]$. 


\subsubsection{Mass Hierarchy}

There are three possible mass splitting for mixing of three flavours of neutrinos,

$$
\begin{aligned}
& \Delta m_{21}^{2}=m_{2}^{2}-m_{1}^{2} \\
& \Delta m_{32}^{2}=m_{3}^{2}-m_{2}^{2} \\
& \Delta m_{31}^{2}=m_{3}^{2}-m_{1}^{2}
\end{aligned}
$$

Where only two of these mass splitting values are independent and are related by,

$$
\Delta m_{31}^{2}=\Delta m_{21}^{2}-\Delta m_{32}^{2}
$$

For $m_{1}<m_{2}$ and $\Delta m_{21}^{2}$ to be positive there are two following possibilities,

- Normal Hierarchy When $\mathrm{m}_{1}$ mass eigenstate will be the lightest one and

$$
m_{1}<m_{2}<m_{3}
$$

- Inverted Hierarchy When $\mathrm{m}_{3}$ mass eigenstate is the lightest one and

$$
m_{3}<m_{1}<m_{2}
$$

There is another mass hierarchy possible when the lightest eigenstate of neutrino is $0.1 \mathrm{eV}$, and when the order of magnitude for masses of neutrinos is larger than the mass splitting values:

- Quasi-Degenerate Hierarchy When the mass difference of state is tiny as compared to the total mass of neutrino eigenstates. In that case we will have,

$$
m_{1} \simeq m_{2} \simeq m_{3} \simeq m_{v}
$$




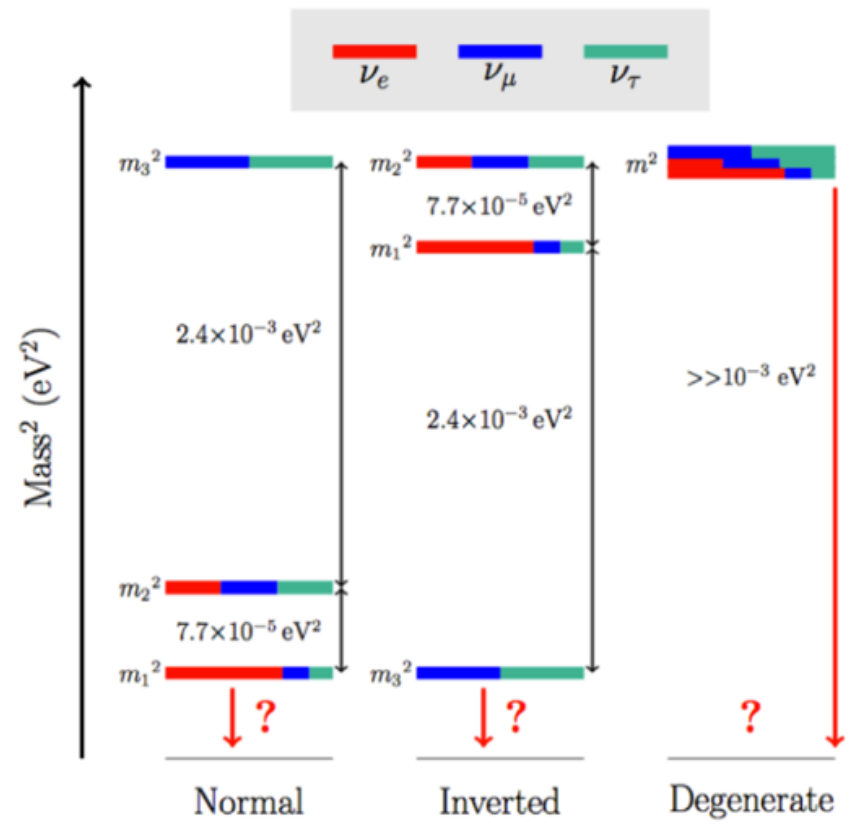

Figure 2.1: Scheme of the normal hierarchy, inverted hierarchy and quasi-degenerate hierarchy, and the color code indicates the flavour $(\mathrm{e}, \mu, \tau)$ present in the mass eigenstates $(v 1, v 2, v 3)$, The mass of the lightest neutrino is unknown [42].

\subsection{Massive Neutrinos in the Standard Model}

The standard model (SM) field theory of elementary particles considers neutrinos as zero mass particles. However, the occurrence of neutrino oscillations, which is already proved experimentally, needs them to be massive. Consequently, there is some required necessary extension in the SM Lagrangian for the implementation of neutrino mass in the model. The scale of masses of neutrino particles is still a big question in physics, and many experiments are working to answer this problem. 


\subsubsection{Dirac Mass}

Neutrinos are half-spin particles which can be described by the Dirac equation,

$$
\mathcal{L}(x)=\bar{\psi}(x)\left(i \gamma^{\mu} \partial_{\mu}-m_{D}\right) \psi(x)
$$

Where $\psi(x)$ is the free neutrino field, and $m_{D}$ is mass of Dirac particle. The Dirac Lagrangian in terms of mass terms can be written as,

$$
\mathcal{L}_{M}=-m_{D}\left(\bar{\psi}_{L} \psi_{R}+\bar{\psi}_{R} \psi_{L}\right)
$$

From the above equation, it can be concluded that the Dirac mass of neutrinos is calculated from the coupling of the left-handed and right-handed spinor of neutrino. However, the weak interaction theory of SM describes only the left-handed neutrinos $v_{L}$ and the righthanded anti-neutrinos $\bar{v}_{R}$.

\subsubsection{Majorana mass}

The neutrino field can be written in terms of two chiral components of neutrinos which gave the Dirac equation is following form

$$
\mathcal{L}=\left(\bar{\psi}_{L}+\bar{\psi}_{R}\right)\left(i \partial_{\mu} \gamma^{\mu}-m_{D}\right)\left(\psi_{L}+\psi_{R}\right)
$$

The Euler-Lagrange equations simplify the above equation as,

$$
\begin{aligned}
& i \partial_{\mu} \gamma^{\mu} \psi_{L}=m_{D} \psi_{R} \\
& i \partial_{\mu} \gamma^{\mu} \psi_{R}=m_{D} \psi_{L}
\end{aligned}
$$

For the massless neutrinos, the above two equations called "Weyl equations" can be decoupled, and the neutrino would be a single chiral field. If the right-handed field of 
neutrinos will be considered as a function of left-handed one, then a Majorana field is defined

$$
\psi=\psi_{L}+\psi_{R}=\psi_{L}+C \bar{\psi}_{L}^{T}=\psi_{L}+\psi_{L}^{C}
$$

This Majorana field is charge invariant, which explains the similarity of particle and antiparticle. Consequently, this field would describe the neutrinos to be their own antiparticles. The Lagrangian mass term for the Majorana field can be written as,

$$
\mathcal{L}_{M}=-\frac{1}{2}\left(\bar{\psi}_{L}^{C} \bar{\psi}_{R}\right) M\left(\begin{array}{c}
\psi_{L} \\
\psi_{R}^{C}
\end{array}\right)+h . c
$$

Where $M=\left(\begin{array}{ll}m_{L} & m_{D} \\ m_{D} & m_{R}\end{array}\right)$, and $m_{L}, m_{R}$ are Majorana mass terms for left-handed fields $\psi_{L}$ and $\psi_{R}^{C}$ respectively, and $m_{D}$ is the Dirac mass term. If neutrinos are Majorana particles, then the above formalism allows a class of right-handed neutrinos with masses different from the left-handed ones. The Seesaw model [43] gives a rational for having very light left-handed neutrinos and very heavy right-handed ones, perhaps as heavy as the Grand Unified mass scale $\left(\sim 10^{16} \mathrm{GeV}\right)[44]$.

\subsubsection{Absolute Neutrino Mass}

The neutrino oscillations observations in atmospheric, solar accelerator and reactor experiments have proved that neutrinos are massive but not given any absolute value for neutrino masses. The ITEP collaboration in Moscow, from the University of Zurich, Los Alamos National Laboratory in New Mexico and from Mainz different physicists, have set an upper limit for the electron neutrino mass with value, $v_{e}$ mass $<2.05 \mathrm{eV}$ [45]. They study the energy spectrum of electrons emitted in beta decay since the non-zero mass of neutrinos caused a truncated spectrum at the end of the beta decay spectrum. The upper limit on the 
mass of the muon neutrino, $v_{\mu}$ mass $<170 \mathrm{keV}$, is concluded by the PSI collaboration [46] at a $90 \%$ confidence interval. They determined this limit by observing the decay of charged pion at rest in the laboratory frame as,

$$
\pi^{+} \rightarrow \mu^{+}+v_{\mu}
$$

The upper limit for tau neutrino $v_{\tau}$ is challenging to be measured. However, ALEPH collaboration observed the decay of $\tau^{-}$into a $v_{\tau}$ as,

$$
\begin{gathered}
\tau^{-} \rightarrow 2 \pi^{-} \pi+v_{\tau} \\
\tau^{-} \rightarrow 3 \pi^{-} 2 \pi+v_{\tau}
\end{gathered}
$$

They set an upper limit on $v_{\tau}$ mass $<18.2 \mathrm{MeV}$ [47].

\subsection{Double Beta Decay}

The discovery of single beta decay played an essential role in neutrino physics with other experiments, which lead to the discovery of neutrino particles. Double beta decay is a rare nuclear decay which is observed in 12 isotopes, with lifetimes of more than $10^{18}$ years. Double beta decay occurs when two neutrons convert to protons by emitting two electrons and two-electron antineutrino particles. It can also occur with the conversion of two protons to neutrons by the emission of two anti-neutrons and absorption of two electrons. In the process of double beta decay, the nucleus changes into an isobar with the emission of other particles as,

$$
(A, Z) \rightarrow(A, Z+2)+2 e^{-}+2 \bar{v}_{e}
$$

The lepton number is conserved in this reaction, and it occurs mostly between eveneven nuclei when single beta decay of the nucleus is not allowed. 


\subsubsection{Neutrinoless Double Beta Decay}

The neutrinoless double beta decay is also a possible occurrence of the beta decay process, which is extremely important in the neutrino physics. This process is forbidden in SM as this mode of decay violates the lepton number by two units. Therefore, if discovered, its study will contribute to physics beyond the SM. In this decay, a nucleus changes as,

$$
(A, Z) \rightarrow(A, Z+2)+2 e^{-}
$$

Which is a two-step process itself as explained by Racah sequence [48],

$$
\begin{gathered}
(A, Z) \rightarrow(A, Z+1)+e^{-}+v_{M} \\
(A, Z+1)+e^{-}+v_{M} \rightarrow(A, Z+2)+2 e^{-}
\end{gathered}
$$

The occurrence of the above equation requires two possibilities which are as follows:

- In the first possibility, the helicity of the process is not conserved as $m_{v}>0$ and transformation is required between $v$ and $\bar{v}$.

- In the second possibility, a right-handed antineutrino is emitted and then annihilated which requires that neutrinos are their own anti-particles.

The simple explanation of Majorana nature of neutrinos (which implies neutrinos to be their own antiparticles) can introduce the idea of existence of some massive virtual light Majorana neutrino $\left(v_{M}\right)$ which got exchanged in the process is given in figure (2.2). 

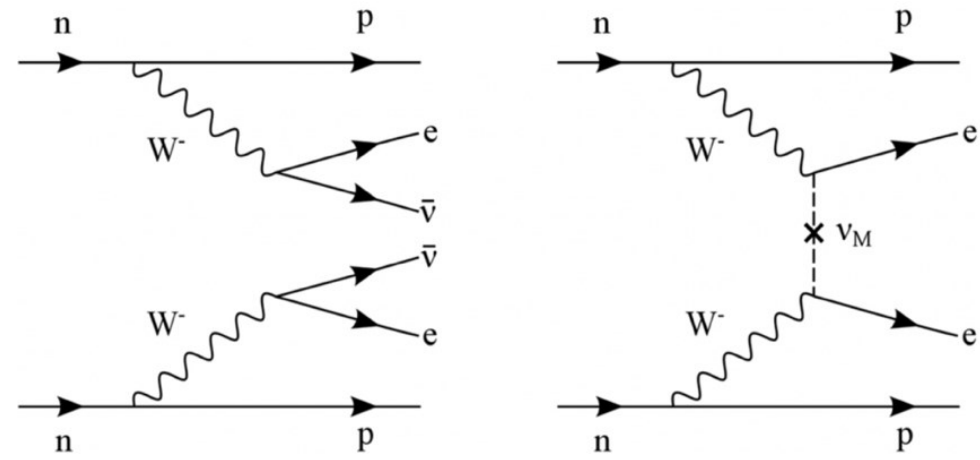

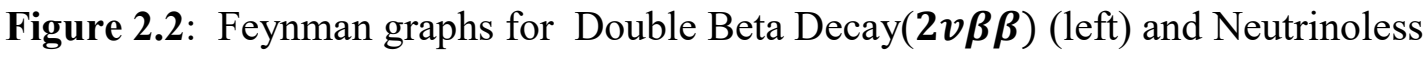
Double Beta Decay $(\mathbf{0 v} \boldsymbol{\beta} \boldsymbol{\beta})$ (right) [49].

The experimental observation of neutrinoless double beta decay process would be extremely important for physics beyond the SM. This observation would contribute to many more questions like $\mathrm{CP}$ violation in leptons and mass hierarchy. The definite evidence of Majorana particle nature of neutrinos would be provided by practical measurement of this decay process, which may also solve the problem of absolute mass of the neutrino particle [31].

\subsubsection{Decay Rates}

\subsubsection{Double beta decay rate}

Fermi's golden rule for a second-order weak decay can be used to calculate the decay rate of $2 v \beta \beta$ process [50] which is given by,

$$
\frac{1}{T_{1 / 2}^{2 v \beta \beta}}=G^{2 v}(Q, Z)\left|M^{2 v}\right|^{2}
$$

Where, $T_{1 / 2}^{2 v \beta \beta}$ is the half-life of process, $M^{2 v}$ is a matrix element, $G^{2 v}$ is the phase space factor which is dependent on $Q$ value, and $\mathrm{Z}$ is the atomic number. 
The particle's energy for $2 v \beta \beta$ case is,

$$
Q=m(A, Z)-m(A, Z+2)-2 m_{e}
$$

and the phase space factor is proportional to $G^{2 v} \propto Q^{7}$.

\subsubsection{Neutrinoless Double Beta Decay Rate}

The decay rate of $0 v \beta \beta$ process calculated from Fermi's golden rule for a second-order weak decay is [50],

$$
\frac{1}{T_{1 / 2}^{0 v \beta \beta}}=G^{0 v}(Q, Z)\left|M^{0 v}\right|^{2}\left(\frac{m_{\beta \beta}}{m_{e}}\right)^{2}
$$

Where $T_{1 / 2}^{0 v \beta \beta}$ is the half-life of process, $M^{0 v}$ is a matrix element, and $G^{0 v}$ is the phase space factor that is dependent on $Q$ value, and $\mathrm{Z}$ atomic number.

The $Q$ value for $0 v \beta \beta$ is,

$$
Q=E_{e 1}+E_{e 2}-2 m_{e}
$$

and phase space factor, $G^{0 v} \propto Q^{5}$.

A complex fundamental quantity appears up in the process of $0 v \beta \beta$ which is called effective Majorana mass given by,

$$
\left\langle m_{\beta \beta}\right\rangle=\sum_{k=1}^{3} V_{e k}^{2} m_{k}
$$

Where V is the PMNS matrix defined in section (2.2.2).

Experiments working on the detection of these decay modes aim to measure the half-life of these decays. Depending on the nuclear model, one can compute the phase space factors and nuclear matrix elements. 
The relation between effective Majorana mass, neutrino mass eigenstate values and parameters of PMNS matrix is as follows,

$$
\begin{aligned}
& \quad\left|m_{\beta \beta}\right|=\left|m_{1} U_{e 1}^{2}+m_{2} U_{e 2}^{2}+m_{3} U_{e 3}^{2}\right| \\
& \quad=\left|\left(m_{1} c_{12}^{2}+m_{2} s_{12}^{2}+e^{-i \phi 21}\right) c_{13}^{2}+m_{3} s_{13}^{2} e^{i\left(\phi_{31}-2 \delta\right)}\right|
\end{aligned}
$$

The predicted $0 v \beta \beta$ effective Majorana mass as a function of lightest neutrino mass is given in (Figure 2:3). There is a quasi-degenerate spectrum which signifies the region when both cases overlap for $0 v \beta \beta$ decay.

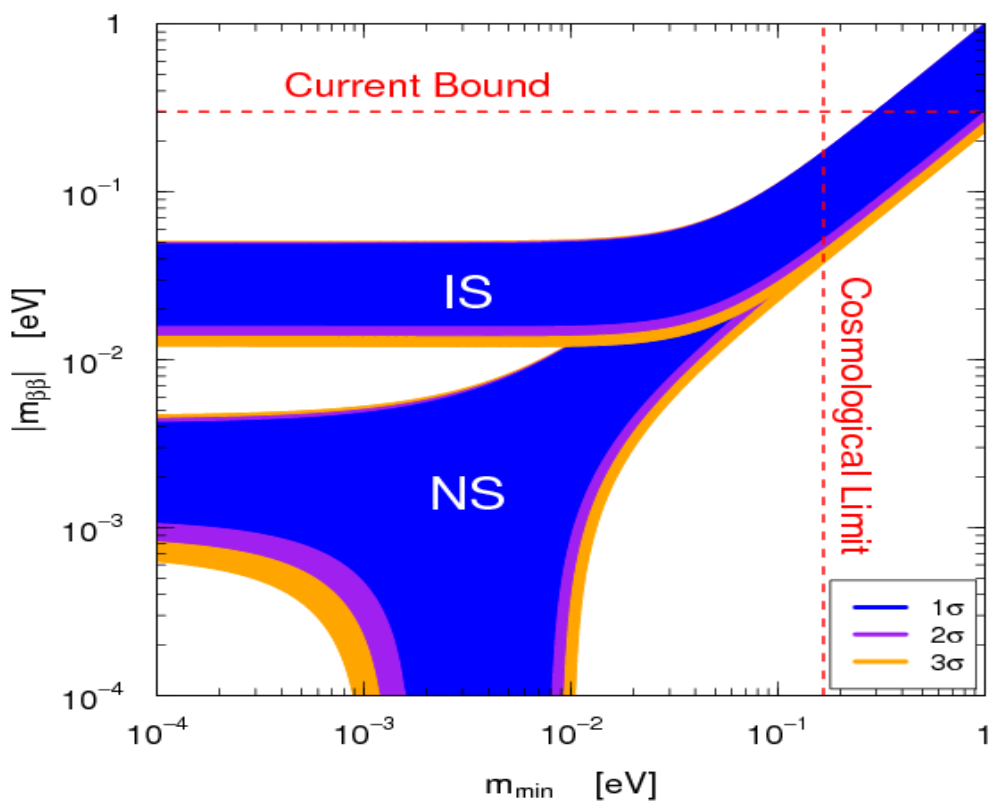

Figure 2.3 Effective Majorana mass as a function of the lightest neutrino mass in the normal and inverted hierarchies. IS denotes the inverted-hierarchy and NS the normal-hierarchy. Modified from [47].

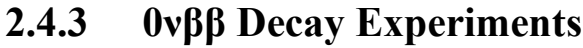

The primary design goal of current experiments looking for new physics is to differentiate between the signal and the effective backgrounds to maintain the detection efficiency high. The energy resolution is the best way to discriminate the $0 v \beta \beta$ signal from the background. 
Therefore, the experiments are looking from some signal which not only is consistent with the $0 v \beta \beta$ decay, but also not matches with any of the background signals. The strategies adopted by present experiments working on to detect the $0 \mathrm{v} \beta \beta$ decay includes the tracking of decay products, which would resolve the two-electron tracks, scintillation and ionization in multiple detection channels to allow the identification of actual physics signal and background. In some experiments with discrete detectors, the energy deposition technique is used for different backgrounds while the background events are rejected by some fiducial volume cuts. In other experiments, the discrimination between signal and background is achieved via machine learning techniques, multidimensional fits, optimizing the discrimination variable, and other methods. Another most important technique to distinguish $0 \mathrm{v} \beta \beta$ decay signal is the event by event identification of the decay daughter, which could be achieved with isotopes such as ${ }^{136} \mathrm{Xe}$ [51]. The ${ }^{136} \mathrm{Xe}$ decay into $\beta \beta$ forms an ionized $\mathrm{Ba}$ daughter product. The nEXO [52,53] and NEXT [54] collaborations are doing their best to identify single Ba ions with high efficiency. Although the implementation of this technique faces many significant challenges, the identification of a $\beta \beta$ decay and capabilities of these experiments to reject the backgrounds are more than important for the development of these experiments to deploy them in the future. For the direct detection of double beta decay signal in experimental physics [51], the detectors are developed from grams to the hundreds of kilograms of the decaying isotope with increased ability to distinguish the physics signal from the backgrounds.

A number of experiments are working currently in various stages of research, development and operation whose aim is to look for $0 v \beta \beta$ in different isotopes. CUORE $\left({ }^{130} \mathrm{Te}\right)$, NEMO $\left({ }^{82} \mathrm{Se}\right)$, PANDA $\left({ }^{136} \mathrm{Xe}\right)$, GERDA $\left({ }^{76} \mathrm{Ge}\right)$, Majorana DEMONSTRATOR 
$\left({ }^{76} \mathrm{Ge}\right)$, AMORE $\left({ }^{100} \mathrm{Mo}\right)$, EXO-200 $\left({ }^{136} \mathrm{Xe}\right), \operatorname{NEXT}\left({ }^{136} \mathrm{Xe}\right)$, and COBRA $\left({ }^{116} \mathrm{Cd}\right)$ are still running to collect data or have completed data collection which would be analyzed further in search of neutrinoless double-beta decay [51]. While there are many future experiments that are in the research phase, such as LEGEND $\left({ }^{76} \mathrm{Ge}\right)$, CUPID $\left({ }^{82} \mathrm{Se} /{ }^{100} \mathrm{Mo} /{ }^{130} \mathrm{Te}\right), \mathrm{nEXO}$ $\left({ }^{136} \mathrm{Xe}\right)$, PandaX-III $\left({ }^{136} \mathrm{Xe}\right)$ and Super NEMO $\left({ }^{82} \mathrm{Se}\right)$, and the construction of many of these experiments has started [51]. Many of these experiments are exploring tonne-level upgrades. Figure (2.4) shows the energy resolution and background index, which are two important detector parameters for some of the past, current and future projects.

\begin{tabular}{|c|c|c|c|c|}
\hline Isotope & $T_{1 / 2}^{0 v}\left(\times 10^{25} y\right)$ & $\langle\mathrm{m} \beta \beta\rangle(\mathrm{eV})$ & Experiment & Ref. \\
\hline${ }^{48} \mathrm{Ca}$ & $>5.8 \times 10^{-3}$ & $<3.5-22$ & ELEGANT-IV & {$[55]$} \\
\hline${ }^{76} \mathrm{Ga}$ & $>8.0$ & $<0.12-0.26$ & GERDA & {$[56]$} \\
\cline { 2 - 5 } & $>1.9$ & $<0.24-0.52$ & Majorana Demonstrator & {$[57]$} \\
\hline${ }^{82} \mathrm{Sa}$ & $>3.6 \times 10^{-2}$ & $<0.89-2.43$ & NEMO-3 & {$[58]$} \\
\hline${ }^{96} \mathrm{Zr}$ & $>9.2 \times 10^{-4}$ & $<7.2-19.5$ & NEMO-3 & {$[59]$} \\
\hline${ }^{100} \mathrm{Mo}$ & $>1.1 \times 10^{-1}$ & $<0.33-0.62$ & NEMO-3 & {$[60]$} \\
\hline${ }^{116} \mathrm{Cd}$ & $>1.0 \times 10^{-2}$ & $<1.4-2.5$ & NEMO-3 & {$[61]$} \\
\hline${ }^{128} \mathrm{Te}$ & $>1.1 \times 10^{-2}$ & - & - & {$[62]$} \\
\hline${ }^{130} \mathrm{Te}$ & $>1.5$ & $<0.11-0.52$ & CUORE & {$[63]$} \\
\hline${ }^{136} \mathrm{Xe}$ & $>10.7$ & $<0.061-0.165$ & KamLAND-Zen & {$[64]$} \\
\cline { 2 - 5 } & $>1.8$ & $<0.15-0.40$ & EXO-200 & {$[65]$} \\
\hline${ }^{150} \mathrm{Nd}$ & $>2.0 \times 10^{-3}$ & $<1.6-5.3$ & NEMO-3 & {$[66]$} \\
\hline
\end{tabular}

Table 2.2: The most updated results of lower limits on $T_{1 / 2}$ and their corresponding upper limits on the effective neutrino mass $m_{\beta \beta}[51]$. 


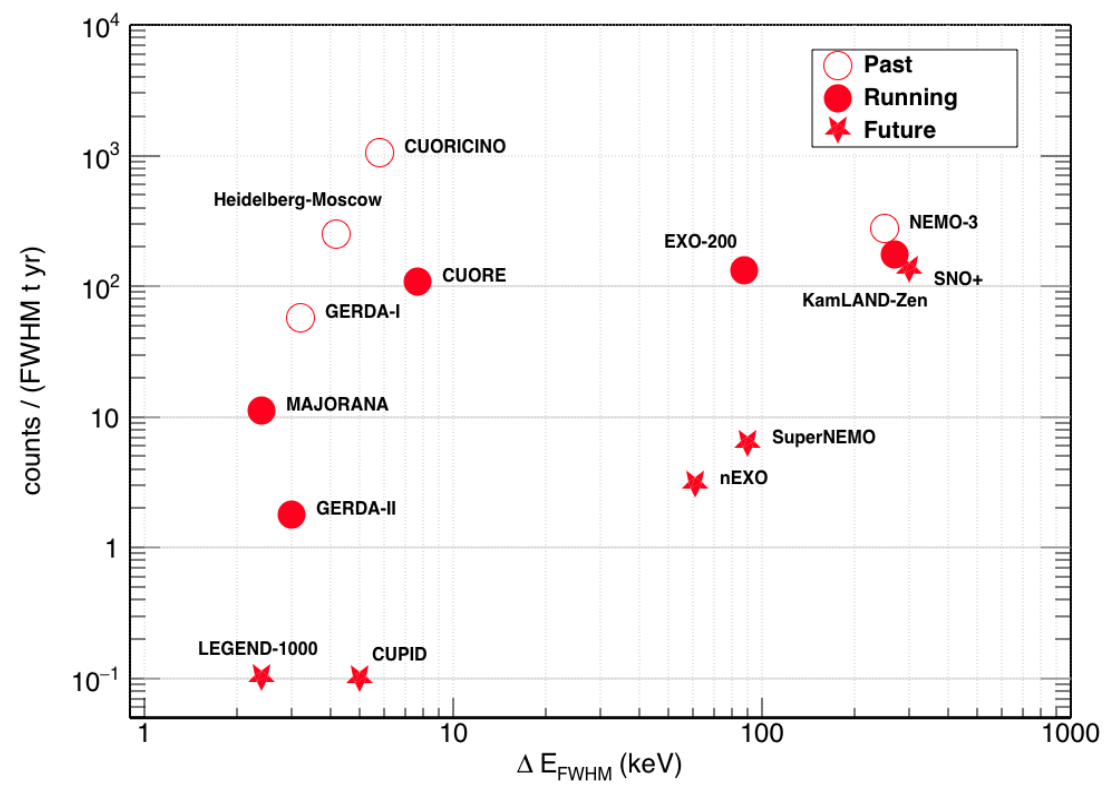

Figure 2.4: The background index as a function of energy resolution for some selected past, current and future projects with ${ }^{76} \mathrm{Ge},{ }^{100} \mathrm{Mo},{ }^{130} \mathrm{Te}$ and ${ }^{136} \mathrm{Xe}$ as target materials [51].

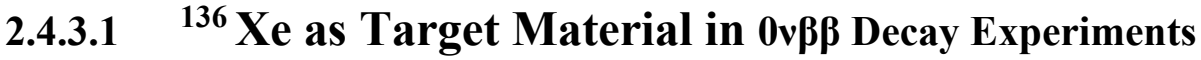

${ }^{136} \mathrm{Xe}$ is considered a very important and attractive target for neutrinoless double beta decay, and other studies since it can be made mostly free of radioactive contamination, has excellent self-shielding from external background, and can be made into detectors of high sensitivity. The most popular detectors for $0 \mathrm{v} \beta \beta$ decay searches working with ${ }^{136} \mathrm{Xe}$ as a target material are as follows:

\section{- The Gotthard TPC}

The Gotthard TPC was located at the Gotthard underground laboratory [67]. It contained the Xenon gas enriched with $68 \%$ of ${ }^{136} \mathrm{Xe}$. This experiment has not observed any excess 
of events beyond the background around, and had a limit of $T_{1 / 2}^{0 v}>4.4 \times 10^{23}$, which was derived for neutrinoless double-beta decay.

\section{- EXO-200}

EXO-200 is a prototype of the Enriched Xenon Observatory (EXO)project, which was in the Waste Isolation Pilot Plant near Carlsbad, NM, USA. It contained the active mass of $110 \mathrm{~kg}$ of Xenon enriched with $80.6 \%$ of ${ }^{136} \mathrm{Xe}$ in a cylindrical single-phase liquid Xenon TPC kept at a temperature of $167 \mathrm{~K}$ [68]. EXO -200 took data in two phases, phase I in 2011, which observed first-time interaction $2 v \beta \beta$ decay in ${ }^{136} \mathrm{Xe}$ [69]. Phase I was ended in early 2014 because of some unrelated fire and radiation release on the experimental site. The experiment was then upgraded to phase (II) from 2016 to 2018. As per the first results from phase (II), the limit for $\left(T_{1 / 2}^{0 v}\right)$ is $1.8 \times 10^{25} \mathrm{y}$ in $90 \%$ C.L. [65].

\section{- nEXO}

$\mathrm{nEXO}$ is the planned research tonne-scale single-phase liquid xenon TPC experiment after the success of EXO-200 [70]. It is planned to fill the TPC with 5000kg of Xenon enriched with $90 \%$ of ${ }^{136} \mathrm{Xe}$. The projected $T_{1 / 2}^{0 v}$ sensitivity is $5.7 \times 10^{27}$ y [7]. At these settings, the $2 v \beta \beta$ decay background will be negligible. It is planned to use the low background materials in the construction of the experiment.

\section{- NEXT}

The neutrino experiment with a xenon TPC (NEXT)is a planned research project with highpressure gas-phase Xenon TPC. NEXT-100 will be deployed with $100 \mathrm{~kg}$ of enriched 
Xenon at pressure of 15 bar located at (LSC) in Spain. It is expected that the $T_{1 / 2}^{0 v}$ sensitivity of NEXT-100 detector will reach up to $2.8 \times 10^{25}$ y $(90 \%$ C.L.) after three years of running the detector $[8]$.

\section{- PandaX-III}

PandaX-III stands for Particle and Astrophysical Xenon Experiment III located at China Jinping underground Laboratory II (CJPL-II). It has a gas phase TPC set at high pressure for the search of $0 v \beta \beta$ decay in ${ }^{136} \mathrm{Xe}$ [51]. The initial first phase of this experiment uses one $200 \mathrm{~kg}$ of Xenon in TPC at a pressure of 10 bar. The future planned phase will be upgraded by five modules to make this experiment a tonne level project. It is planned to project the $T_{1 / 2}^{0 v}$ sensitivity to $10^{26} \mathrm{y}$ in three-year data from this experiment [71].

\section{- KamLAND-Zen and KamLAND2-Zen}

KamLAND stands for Kamioka Liquid scintillator Antineutrino Detector located at Kamioka observatory in Gifu prefecture in Japan. The word Zen means Zero neutrino searches for $0 v \beta \beta$ decay in ${ }^{136} \mathrm{Xe}$. This experiment uses the different amounts of ${ }^{136} \mathrm{Xe}$ enriched to $90 \%$. The lower limit for sensitivity $T_{1 / 2}^{0 v}$ obtained in phase II of the KamLAND-Zen 400 experiment using $400 \mathrm{~kg}$ of $\mathrm{Xe}$ is $1.07 \times 10^{26} \mathrm{y}(90 \%$ C.L.) with a median sensitivity of 5.6 $\times 10^{25} \mathrm{y}[64]$. The upgraded planned generation of the KamLAND Zen experiment is the KamLAND2-Zen experiment. It is planned to use a tonne of enriched Xenon with improvements to reduce the $2 v \beta \beta$ decay backgrounds. A factor of two improvement in energy resolution from the new version of the experiment is expected to result in a sensitivity of $\sim 2 \times 10^{27}$ with five years running of the KamLAND2-Zen experiment. 
These experiments prove the importance use of ${ }^{136} \mathrm{Xe}$ for the search for double beta decay. Many groups [5-8] are in the process of constructing or proposing the xenon based detectors, and the cost of those proposals is dominated by the high cost of xenon and the requirement of isotopic enrichment to limit the total xenon to be used. At the time of writing, the sole supplier is a Russian group who use a centrifuge system. The current cost is estimated to be $\$ 10-20 \mathrm{M} /$ tonne. For the next generation double-beta experiment, up to 5 tonnes are required, making this by far the most expensive component in the experiment. Distillation is gaining popularity as an economic separation technique, and it was the technique used to produce heavy water for SNO experiment. This study aims to explore the feasibility of xenon separation on a tonne scale using distillation as we will see in the next chapters. 


\section{Chapter 3: Operation of a still}

\subsection{Distillation Conception}

The phenomena of purifying the liquid mixtures by boiling the substance to make into vapors and condensing them back to the original liquid is called distillation. There are different types of distillation processes used in industries such as petroleum industry, petrochemical, coal tar and other chemical and pharmaceutical industries to purify the individual components of a substance into pure form. More commonly, the distillation process is used for removal of salt from the seawater to get chemically pure water. In the basic principle of working of some distillation process, a material to be purified is put in column. When the material with several miscible liquids is heated, the components with low boiling points gets converted to gas phase first then the components with higher boiling points. The distillation process removes these vapors and condenses it back to another different receiving flask in liquid phase. Therefore, the different components of liquid are collected in separated flasks by collecting the condensed vapors as fractions. The remained behind non volatile residue is also considered as some others impurity present in the material.

\subsection{Isotope Separation by Distillation}

Isotopes of the different element existing naturally have different uses in the fields of the study for example hydrology, geology, and medicine. These applications include the various techniques like to find the isotopic presence of a material under investigation or tracing the isotopes of some material in one part of system to other. There are many techniques 
used in these fields which results the raw information about isotope which got further used in various experiments of biology and the medical sciences. The isotopic substances are not various in the chemical and physical properties, but the mass and thermodynamic properties could also be used to detach them [72].The isotope separation methods are generally based on the isotopic effect of different compounds whose isotopes exists and these isotopic [73] effects are mainly because of the different nuclear properties of the isotopes of the material. Chemical exchange process, diffusion-based separation, laser separation etc. are some experimental methods used for the separation of different isotopes of some compound [74].These process depend upon the chemical properties of the compound, includes the other cost of the procedure and on the different applications that use the different concentrations. The distillation method is commonly used for the isotope separation of elements like oxygen, carbon, nitrogen or boron. This idea works on the relatively large difference of mass between the isotopes of these light elements. The distillation process of isotope separation is based on the vapor pressure isotope effect theoretically [73].

Distillation process are most commonly used techniques for the separation of isotopes light elements. The operation of these techniques based on the relative volatility notion which is the measure of the vapor pressure of any mixture. Distillation is mostly carried in two types of columns. Tray columns in which ratios of liquid flow rate to vapor flow rates is high and the packed column where the diameter of the columns is small and they can be used in conditions where pressure drop is low, in handling of the corrosive materials [75],[76]. 


\subsection{Need for VPIE pressure differences for different isotopes}

In a noble fluid, different isotopes of the same chemical species can have slightly different vapour pressures. In the vapour phase, there is little interaction beyond hard sphere scattering, between the atoms. In the liquid, atoms interact with their neighbours constantly, through a Van Der Waals force and this gives rise to rotational and vibrational states. The energies of these depend on mass and thus the density of states for a given energy are slightly higher for heavy isotopes by comparison with lighter ones. This results in the heavier isotopes having slightly lower vapour pressures.

There were no experimental VPIE data for Xe until 1942 when Clusisus tried a direct measurement of the vapour pressure which failed somehow[1]. There was distillation [3]work done by Grigor'ev. In that distillation measurement the accuracy was very small as compared to differential pressure VPIE values. There are two different theoretical calculations discussed by [1]. Both calculated the $\ln \left(f_{c} / f_{v}\right)$ for Xe where $\mathrm{f}$ is reduced partition function ratio for condensed phase " $\mathrm{C}$ " and vapor phase "V". Lopes [77] shows that,

$$
\ln \frac{f_{c}}{f_{v}}=\ln \frac{p_{i}}{p}
$$

Where $p_{i}$ is the vapour pressure for isotope $\mathrm{i}$, and $p$ is the mean vapour pressure.

As the VPIE are very small numbers,

$$
\ln \frac{f_{c}}{f_{v}}=P V I E
$$

The treatment of Lopes [77] yields the value $\ln \left(f_{c} / f_{v}\right)=2.7 \times 10^{-4}$ for ${ }^{130} \mathrm{Xe}$ to ${ }^{136} \mathrm{X}$. 
The other calculation done by Chialvo and Horita [78] derived a similar result. Their Calculations for the isotope pair from ${ }^{132} \mathrm{Xe}$ to ${ }^{136} \mathrm{Xe}$ yield the result $2.1 \times 10^{-4}$ for the VPIE. Correcting them to a mass difference of 6 Atoms Tew gets VPIE $=3.2 \times 10^{-4}$ in good agreement with Lopes.

\subsection{Distillation process for this experiment}

Large-scale distillation with $100 \%$ reflux has been used in this experiment to separate the isotopes of Argon, Krypton and Xenon. The distillation process is based on the fact that the isotopes have slightly different vapour pressure according to their masses. The greater difference in the vapour pressure, easier it would be to separate the isotopes. In the distillation column used in experiment, there is a condenser fixed nearby at the top of the still which cools the noble gas injected from the top. The gas then flows through the special packing material in the still, cools and falls down into the reservoir. A boiler is installed at the base to heat the liquid mixture. As the liquid mixture gets heated, some of its molecules gain kinetic energy and escape the intermolecular forces to get evaporated to the gaseous state. The light isotopes have low boiling points and they get evaporate in large number than the heavier isotopes at any given temperature. The vapor passes through the packing material and partly recondenses into the liquid at the surface of packing material. The liquid then again re-evaporates and condenses in layers of packing material with the enrichment per layer of isotope remaining constant. The still has 100\% reflux implying that there is no overhead product, and there is no material taken of the bottom . Cycle of evaporation and condensation continues until an until equilibrium is attained through the column with high abundance of lighter isotopes at the top and heavier isotopes at the bottom of the still. The 
separated isotopes can be extracted from the column. Figure (3.1) shows the general scheme of this process.

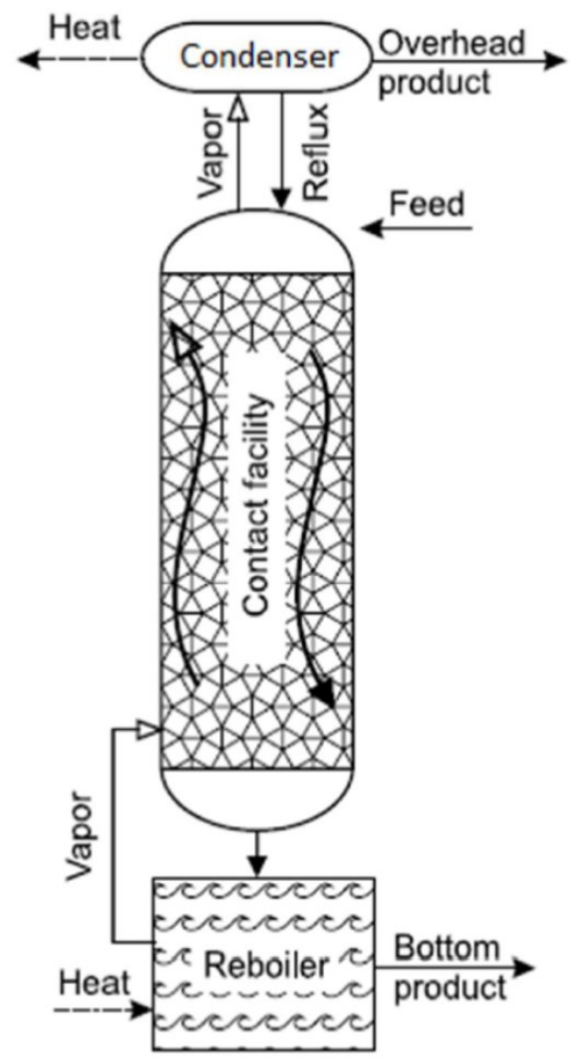

Figure 3.1: Basic diagram of distillation process used in the experiment [79].

\subsubsection{Height Equivalent Theoretical Plate (HETP)}

The distillation column can also be splitted into different sections which are separated by different plates and holes can be used in place of packing materials. The vapour of liquid mixture will rise through the holes and condenses on the plate. Once the equilibrium is reached, the lightweight isotopes will likely to be collected on the top plates. Thus, the use of more sections will result in the better separation of the molecules. The separation of tray heights was determined experimentally, and total height of the column was designed based 
on the enrichment per tray. The packing materials works some way more efficiently for narrow columns, and the equilibrium is attained quickly in these columns in shorter distance. The HETP (Height Equivalent Theoretical Plate ) is the height of packing material needed for the vapour and liquid flows to be in equilibrium, representing a hypothetical plate separation distance.

For a still separation two components (light and heaver) the separation is defined by,

$$
S=\frac{\left(C_{\text {light }} / C_{\text {heavy }}\right)_{\text {Top }}}{\left(C_{\text {light }} / C_{\text {heavy }}\right)_{\text {Bottom }}}
$$

Where, $\mathrm{C}$ is the concentration of the light/heavy component with measurements made either at the Top or Bottom of the still.

The relative volatility $(\alpha)$ is defined as, $\alpha=$ (vapour pressure of the light component) / (vapour pressure of heavy component).

$$
\alpha=1+\delta P / P_{i}
$$

The number of theoretical plates, $\mathrm{N}$ is given by,

$$
N=\frac{H}{H E T P}
$$

Where, $\mathrm{H}$ is the total column height, and HETP is Height Equivalent Theoretical

Plate.

Then, Fenske's formula $[73,80]$ for a still operating with constant relative volatility and with total reflux is ,

$$
H E T P=\frac{H}{N}=\frac{H \ln (\alpha)}{\ln (s)}
$$

From this we can see that,

$$
s=\alpha^{N}
$$

For this thesis, these formulas are generalized to a multi component mixture as, 


$$
\begin{gathered}
S_{i}=\frac{\left(C_{i} / C_{\text {Strong }}\right)_{\mathrm{Top}}}{\left(C_{i} / C_{\text {Strong }}\right)_{\text {Bottom }}} \\
S_{i}=\alpha_{i}^{N}
\end{gathered}
$$

and,

$$
H E T P=H \frac{\operatorname{Ln}(\alpha)}{\operatorname{Ln}(S)}
$$

This formula is used to calculate HETP for the column using the measured separation for argon isotopes and the known value for $\alpha$. The equation is then inverted to determine the values of $\alpha$ for the krypton and xenon isotopes, from the measured separations. 


\section{Chapter 4: Description of the Carleton Experiment}

\subsection{Overview of Experiment Performed}

A noble liquid distillation test plant has been constructed at Carleton University to measure the separation capability of such a still for producing enriched ${ }^{136} \mathrm{Xe}$ for searches for neutrinoless double beta decay. While data exist for the dependence of vapour pressure on isotopic number for a number of noble systems (including argon and krypton), no such data exist for xenon. Distillation separation relies on the small difference in vapour pressure of different isotopes, most pronounced near the triple value. The plan for our system is to characterize the still using argon, to test the mass extrapolation by studying krypton and then finally to do a full study of the separation of xenon isotopes to get credible data that could be used to assess the economic feasibility of producing ${ }^{136} \mathrm{Xe}$ using a distillation.

The test system consists of a packed column $2.5 \mathrm{~cm}$ in diameter and $183 \mathrm{~cm}$ high. A cold head at the top can provide cooling at controllable power to condense the gas while a heater at the bottom in the re-boiler will evaporate the liquid. Thus, there is a liquidvapour counter flow in the column. The packing used is Propak of $1 / 8$ ". Vapour samples can be taken from the top of the stack or from the bottom and sent to a mass spectrometer for isotopic abundance measurement. The mass spectrometer is a quadrupole of $19 \mathrm{~mm}$ bore operating at $1.2 \mathrm{MHz}$. The column is surrounded by super insulation and is located in a vacuum jacket. A data set consists of a measure of the mass distribution at the top and then at the bottom. Measurements were made at a pressure of $100 \mathrm{kPa}$ and the same molar vapour flow rate was used in each case. Figure (4.1) shows a photo of the system. 


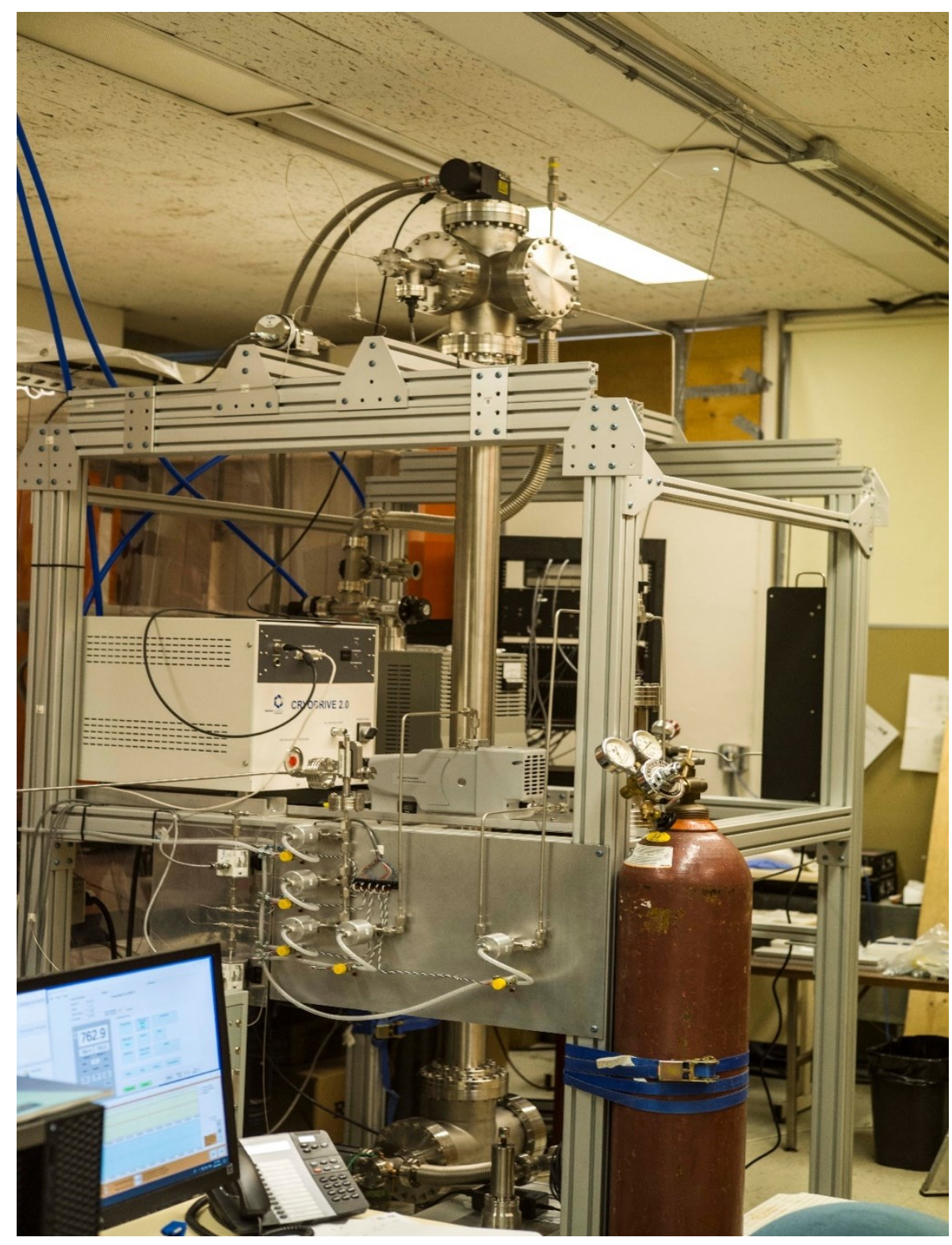

Figure 4.1: Distillation system at Carleton is $2 \mathrm{~m}$ long. The condenser (operating dawn 200C) is located in the top 8" cross while the reboiler is in the bottom cross. The packed column fills the space between, inside a vacuum jacket. This has been run with argon, krypton, and xenon gasses. 


\subsection{Experiment setup}

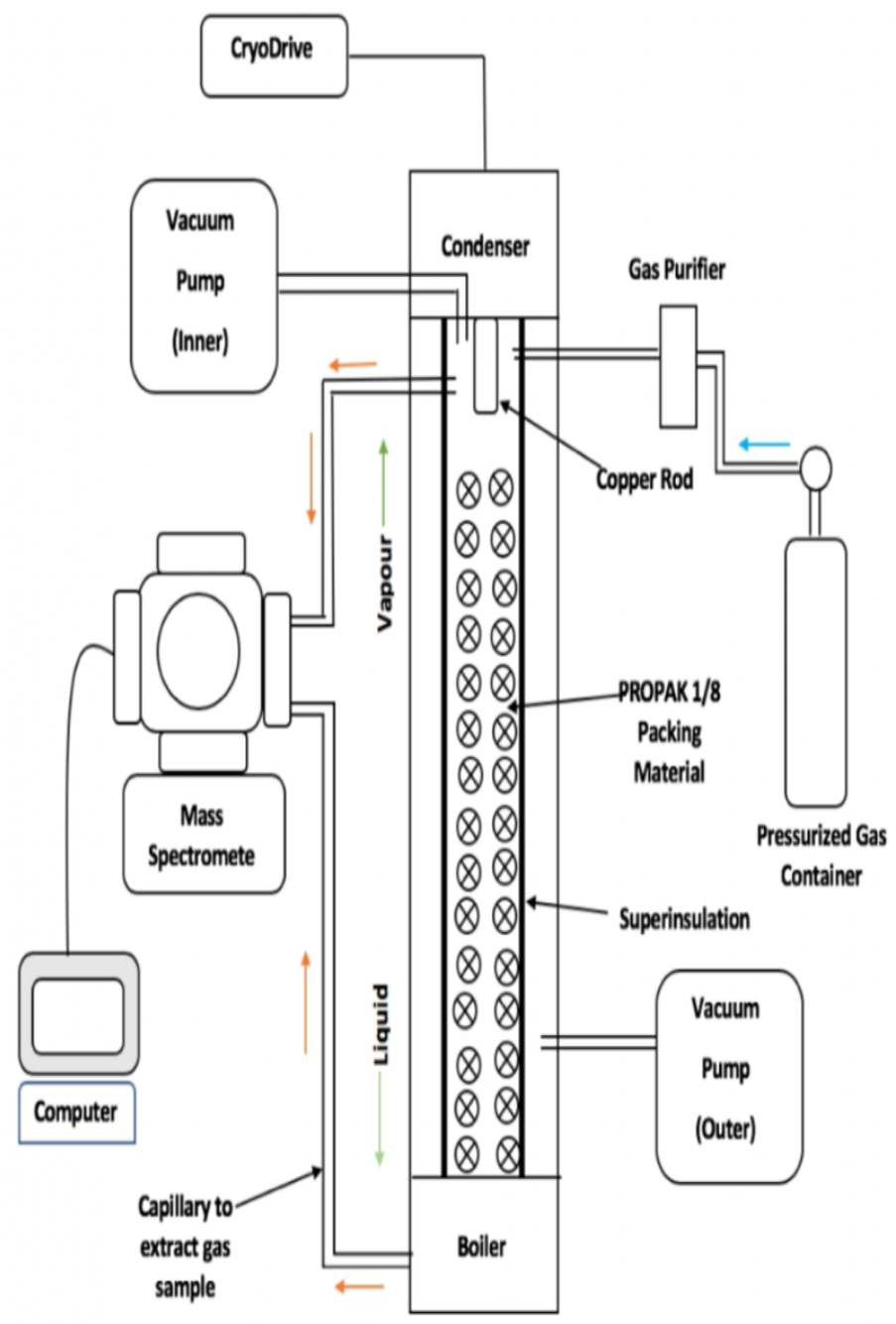

Figure 4.2: Experimental setup showing gas entering the top of the still, with samples extracted from the top $\&$ bottom to be sent to the mass spectrum [81]. 


\subsection{Equipment Design}

\subsubsection{The distillation column}

The column for the distillation is assembled using sections of 1.25 " stainless tubing (1.12" or $27.4 \mathrm{~mm}$ ID) each 6 feet $(1.76 \mathrm{~m})$ in length and fitted with 2.75 " conflat connections at the ends. This inner tube is wrapped with superinsulation and located inside a 4" tube, also supplied in 6' lengths and sealed with 6" conflat flanges. The column is filled with a special stainless packing material (PROPAK 1/8") [82]. A screen just above the boiler supports the packing. The manufacturer does not recommend intermediate supports in a column this small. A condenser at the top (Oxford Cryosystems 40-60 W) will liquefy the xenon vapour and a heater at the bottom will vapourize the liquid xenon. The boiler is designed to produce mixing of the liquid volume. Two capillary tubes will allow the vapour at the top and bottom of the packed column to be extracted and sent to a quadrupole mass spectrometer for isotopic analysis. The column is modular in 6-foot lengths. Initially a single length will be used to commission all systems at Carleton. If the system performs to our satisfaction, the system will be transported to SNOLAB where it will be installed in one of the high halls. Seven additional columns lengths will be added to give a total column height of 48 feet.

Two vacuum systems are provided, one pumps continuously on the space between the inner and outer pipes while the other evacuates the packed column prior to a measurement. Both units are Varian Task V70LP and consist of a turbo pump backed by a scroll pump. The pressure in the packed column is brought to below $10^{-7}$ torr prior to adding the noble gas while the outer vacuum is typically $10^{-6}$ torr. 


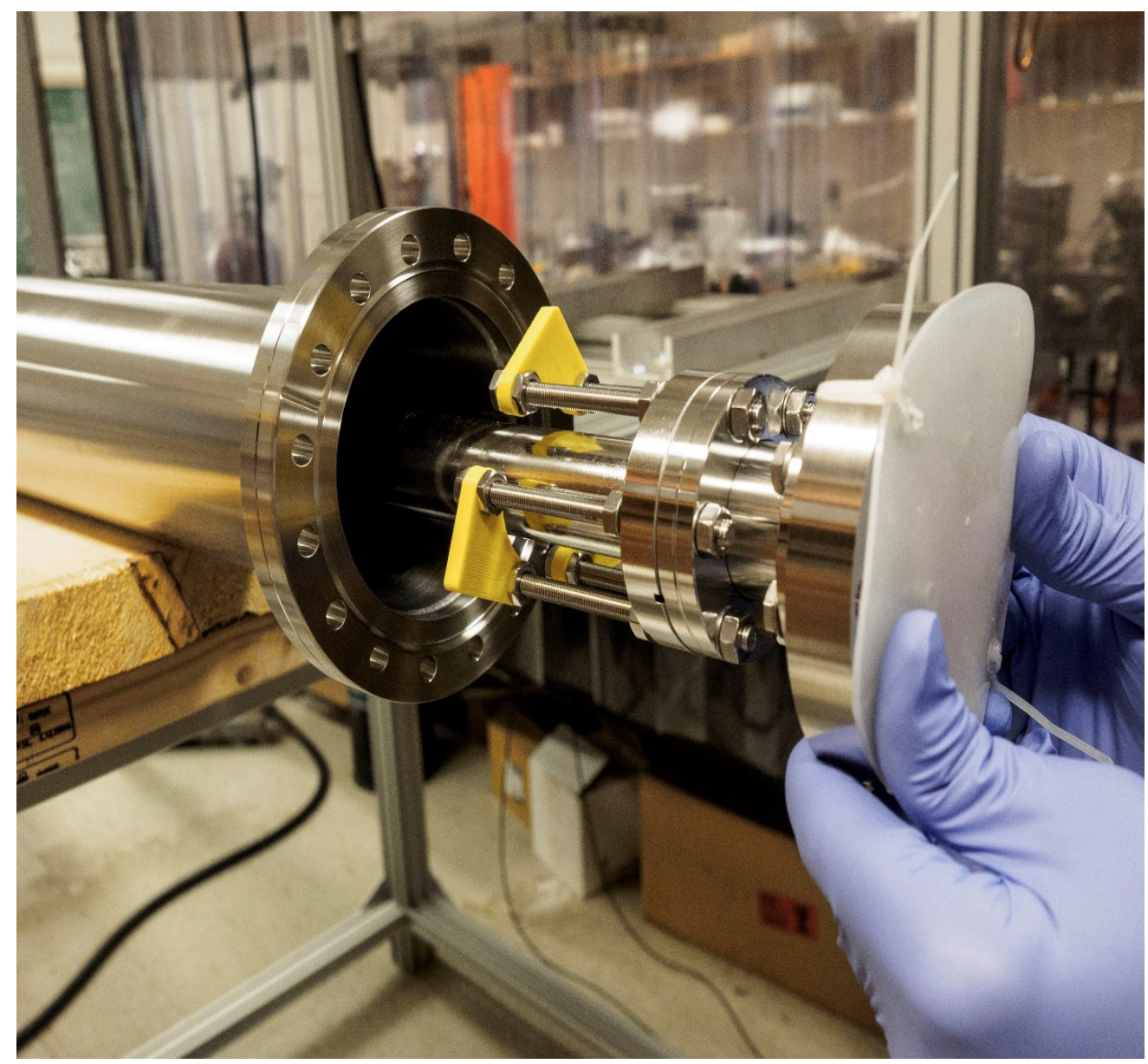

Figure 4.3: Assembly of the inner and outer pipes showing the spacers. Super insulation on the inner pipe starts just above the region shown.

\subsubsection{Stainless Steel Boiler (Made custom at Carleton University)}

There is a boiler at the base of the distillery that heats the liquid reservoir in order for it to evaporate up through the column. The boiler is made of a 4.5 " conflat $1 / 2$ nipple (about 2.5" diameter pipe). Heating takes place in the side arms to ensure mixing of the liquid. Two sample lines are seen allowing sampling of the vapour above the boiler or below the liquid. The sample lines are $1 \mathrm{~mm}$ ID capillary tubes. 


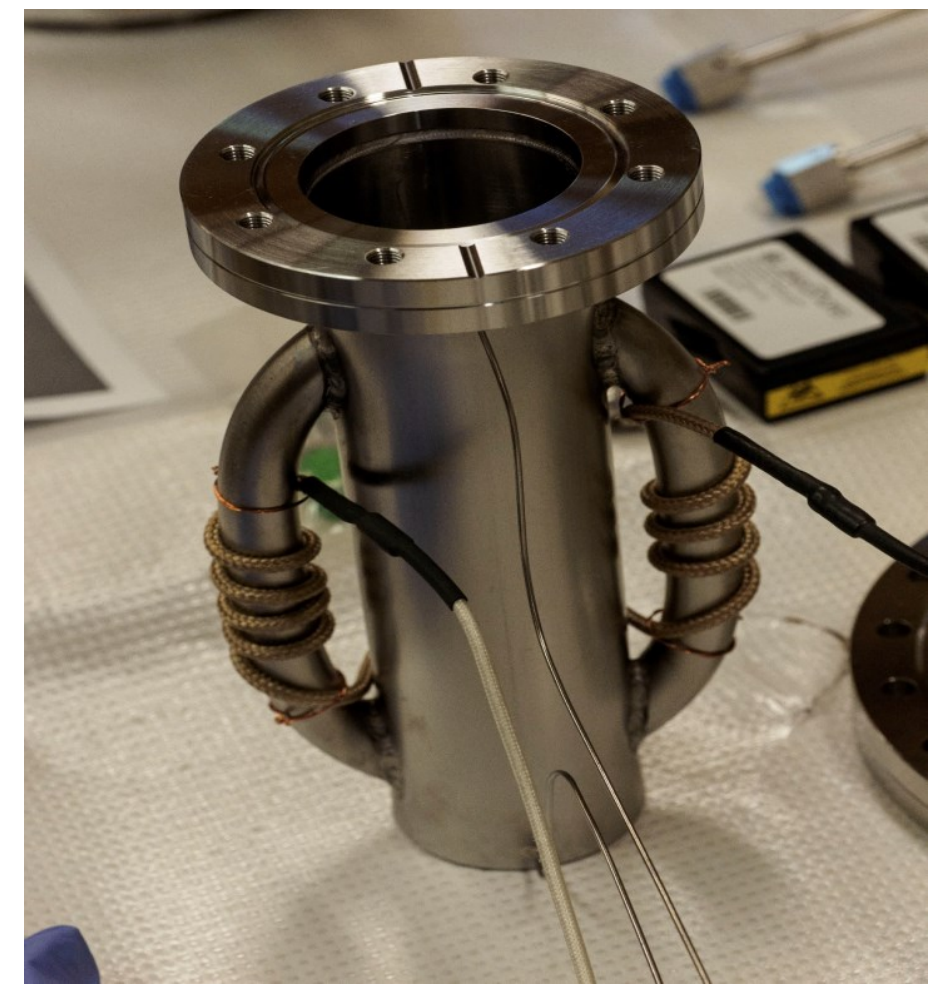

Figure 4.4: The Boiler.

\subsubsection{Oxford Cryosystems CryoDrive 2.0 (40-60W)}

A condenser at the top (Oxford Cryosystems 40-60 W) will liquefy the xenon vapour and a heater at the bottom will vapourize the liquid xenon. There is a copper rod that sticks down into the distillery from the top and the condenser makes this rod really cold. When the gas enters the distillery, it condensed onto the rod, liquefies and drips down onto the packing material and pools into a reservoir at the base of the column. For control, the refrigerator cooling power is set. Then, the heating power is controlled to maintain a fixed pressure. All measurements performed at $100 \mathrm{KPa}$ absolute pressure. 


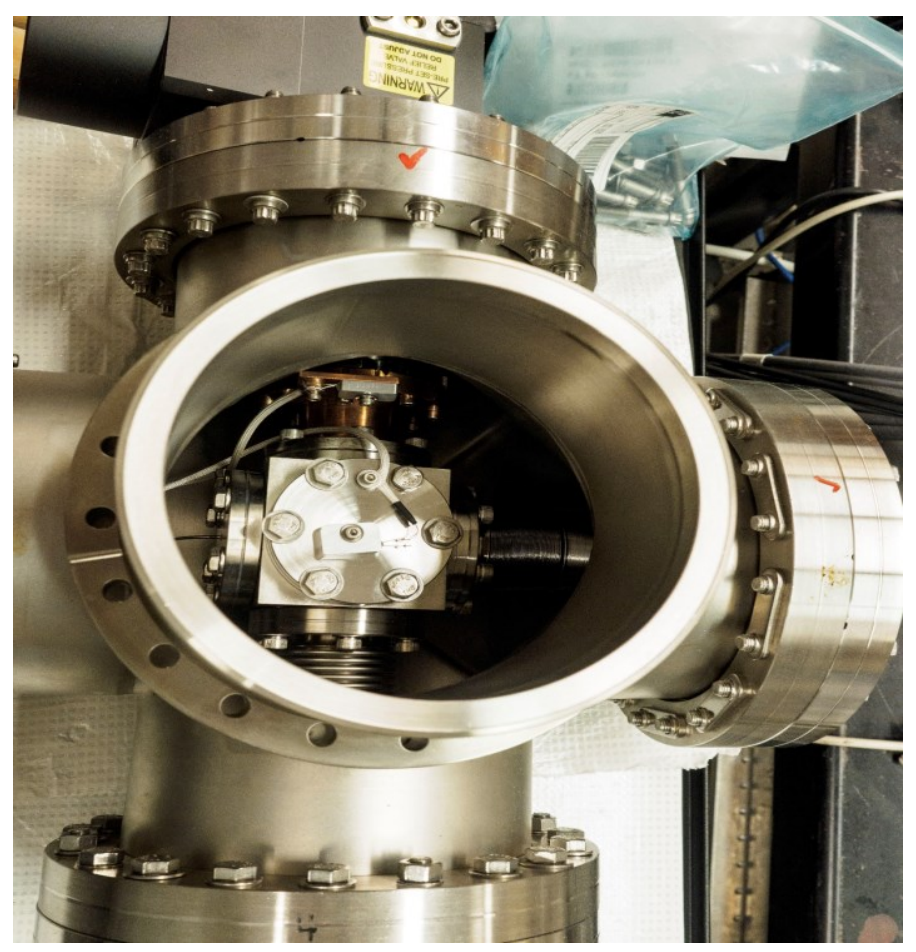

Figure 4.5: Condenser region. The copper cold finger is shown at the top entering a 2.75" cube which forms the condenser. The packed bed is located below the lower bellows. Gas connections to the cube enter through the bellows at the right. A Pt thermometer is located in the center of the flange facing you.

\subsubsection{Sampling systems.}

Gas can be extracted from the top of the still, and from the bottom of the stack. Sampled vapour is transported in fine capillary tubes to a quadrupole mass spectrometer based on an EXTREL MAX-500 unit. This unit has a $19 \mathrm{~mm}$ bore and a quoted resolution of 1:3000. Separation of the isotopes is very clean. There is provision for pump out of the capillary system prior to each measurement to ensure the sample measured is representative of the material in the still at the time of measurement. The quadrupole operates in a mode where 
the ion detector reports a current as the mass is scanned. The measured currents are averaged over many mass scans to produce a final spectrum. It is thus not a counting measurement and run-to-run fluctuations must be determined by making several runs and calculating the variances.

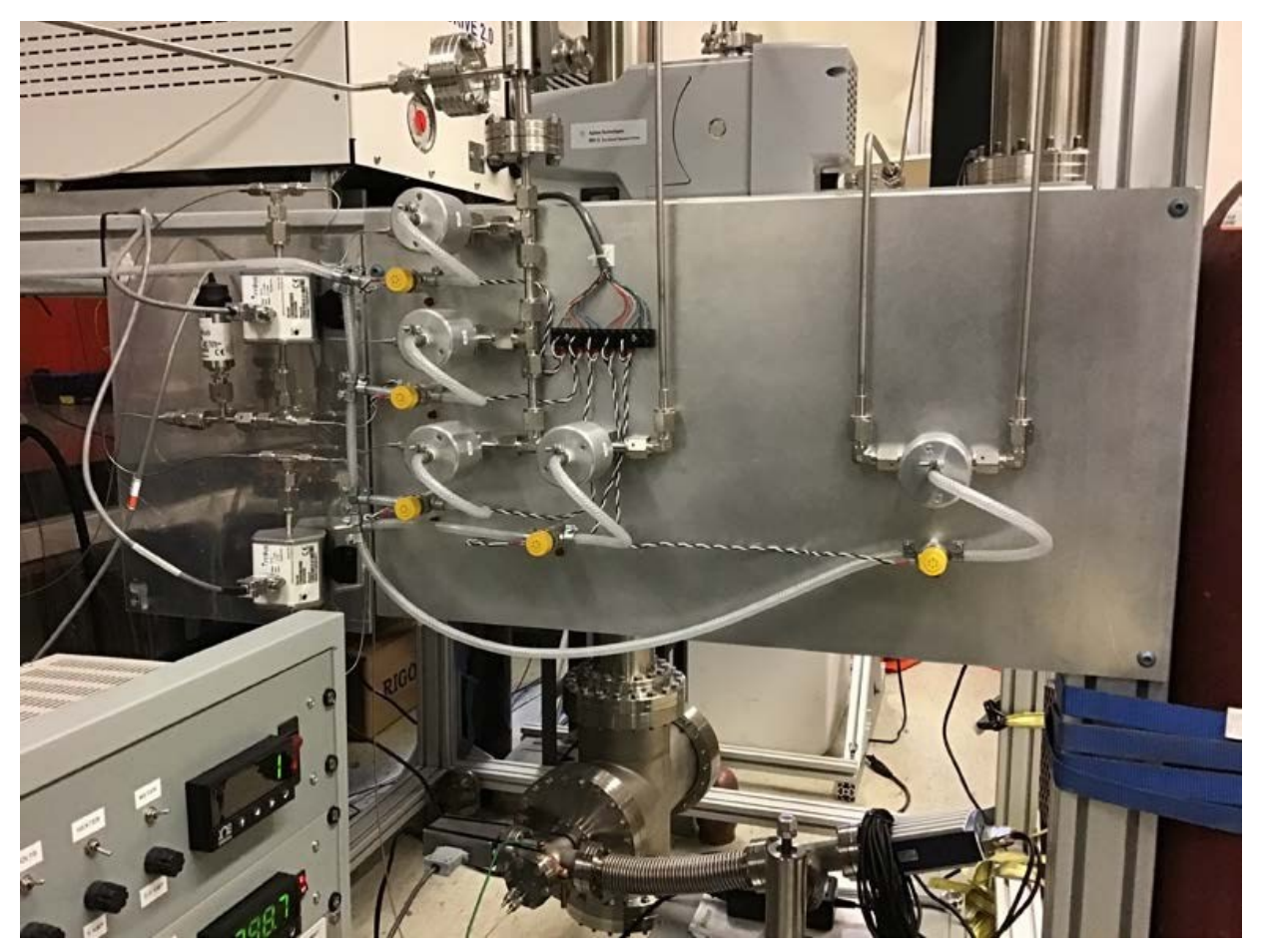

Figure 4.6: Gas sampling control panel installed at lower level attached to tower.

\subsubsection{SAES PS3-MT3 Gas Purifier Getter}

The Getter is a filter that purifies the gas. The noble gas goes through before entering the distillery in order to remove any traces of other molecules that are not the noble gas. The system is PS3-MT-3. This is rated for 5 SLPM flow rates but the supplier suggests keeping the flow below 1 SLPM for xenon. This is not a serious limitation for us. 


\subsubsection{PROPAK 1/8" Stainless Distillation Packing}

Pro-Pak [82] is a stainless-steel ribbon perforated to gives a very high surface area bent into a U shape. When the gas evaporates from the liquid pool at the bottom of the distillery, it rises through the packing material and partly condenses on the surface. Then equilibrium is reached for every HETP (Height Equivalent Theoretical Plate).

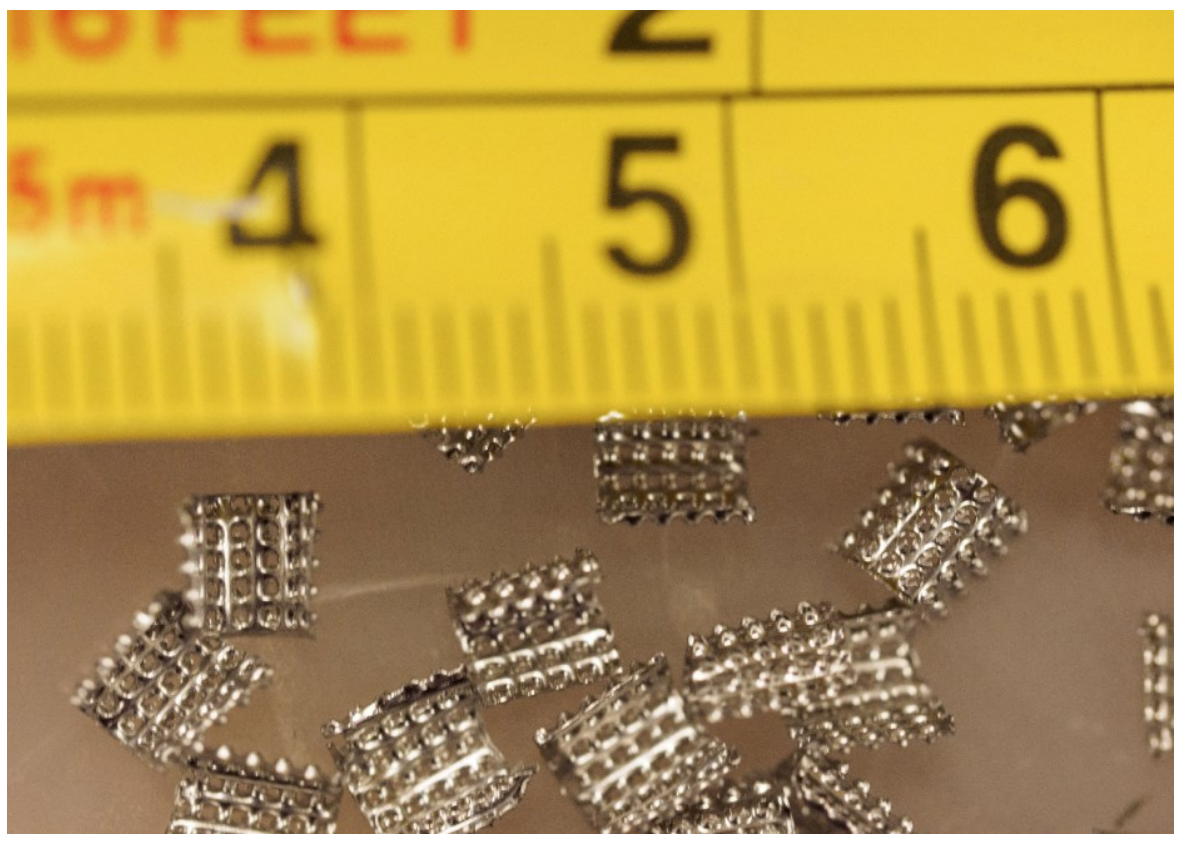

Figure 4.7: Propak 1/8 which gives a very high surface area bent into a $U$ shape [82].

\subsubsection{Gas system}

For operation with xenon a cryogenic recovery system is used to supply and recover this expensive commodity. For the studies with argon and krypton, this recovery system is not used. 

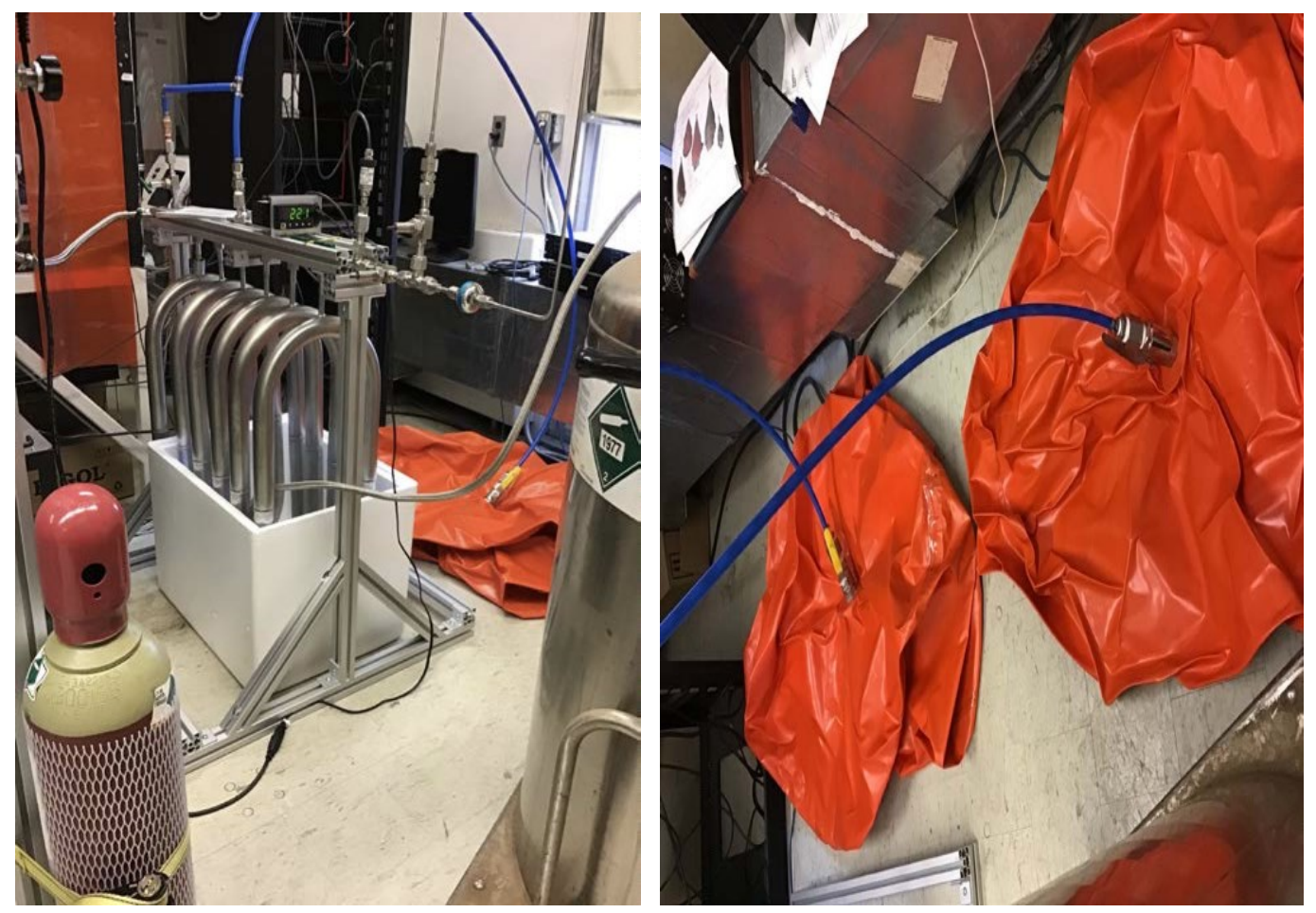

Figure 4.8: Xenon recovery system installed at lower level and Emergency xenon recovery balloons installed at lower level.

\subsubsection{Pressure Sensor and Temperature Sensor}

There are pressure and temperature sensors located at the top of the distillery and at the top/bottom of the reservoir. The pressure sensor is connected to sample lines. The top pressure sensor is precision capacitance manometer with 1000 torr full scale and an accuracy of $0.125 \%$. We measured the total pressure at the top gas vapour phase. We measure the differential pressure between the top and the bottom of the column, and between the top and bottom of the boiler. The first differential pressure tells you the pressure drop cross the column and the second one tells you how much liquid is in the boiler. 


\subsubsection{Quadrupole Mass Spectrometer}

This is the mass spectrometer used to determine the relative abundance of the isotopes from the gas extracted from either the top or bottom of the distillery. The intensity of different ions from isotope was measured using a Quadruple mass spectrometer. This apparatus is common among many residual gas analyses because of its high stability and mass resolution. The working of QMS is not very simple from the magnetic sector design, which includes some specific mathematical calculations, and it is elegant. A QMS is made of following different parts which are ionizer, an ion accelerator, and a mass filter which function together to give the mass spectra of ions.

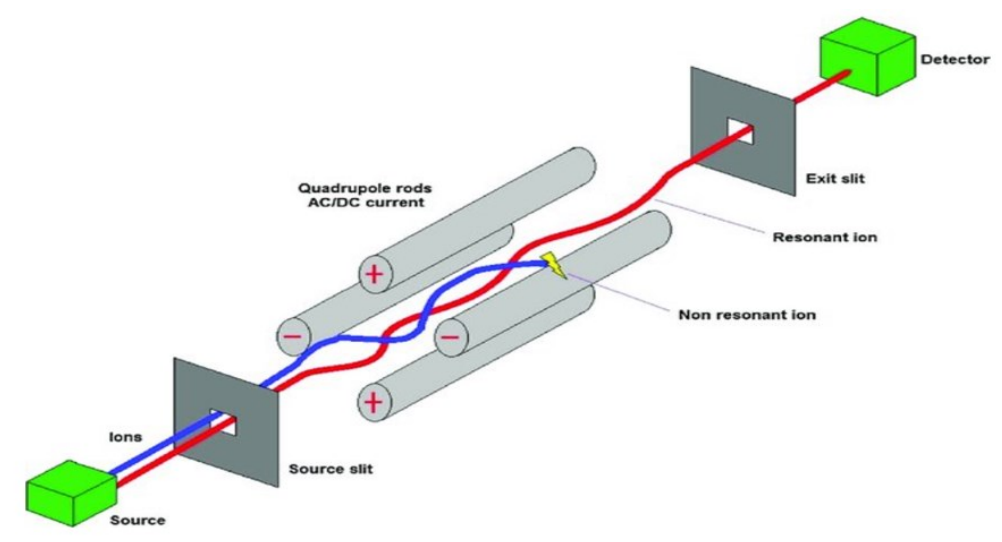

Figure 4.9: Diagram of Quadrupole mass spectrometer [83].

\subsubsection{Quadrupole Mass Filter}

The qualities of quadrupole mass filter (QMF) which make them popular mass spectrometers are their small size, lightweight, cheaper and fast scan times (less than $100 \mathrm{~ms}$ ). They are capable of being operated at relatively high pressure $\left(5 \times 10^{-5}\right.$ torr $)$ and hence used commonly in GC applications and sometimes in LC systems. A quadrupole mass filter 
(QMF) is generally made of four parallel metal rods, two of which are connected to DC voltage $(\mathrm{U})$ superimposed with a radio frequency $\left(\mathrm{V}_{0}\right)$. The other two metallic rods are then placed diagonally to the first pair of rods, which are then connected to AC voltage such that the electrode pairs would be out of phase with each other. The resulted electric field of these rods' pairs will cause the inlet ions entering the Quadruple to travel in the oscillatory motion in the $\mathrm{X}-\mathrm{Y}$ plan and forward motion in the $\mathrm{Z}$ direction. $\mathrm{DC}$ and $\mathrm{RF}$ voltages applied to the poles cause most ions to be deflected out of the beam. Only these ions having the selected mass have stable trajectories at the operated DC and RF can pass the length of the filter and finally reach the detector.

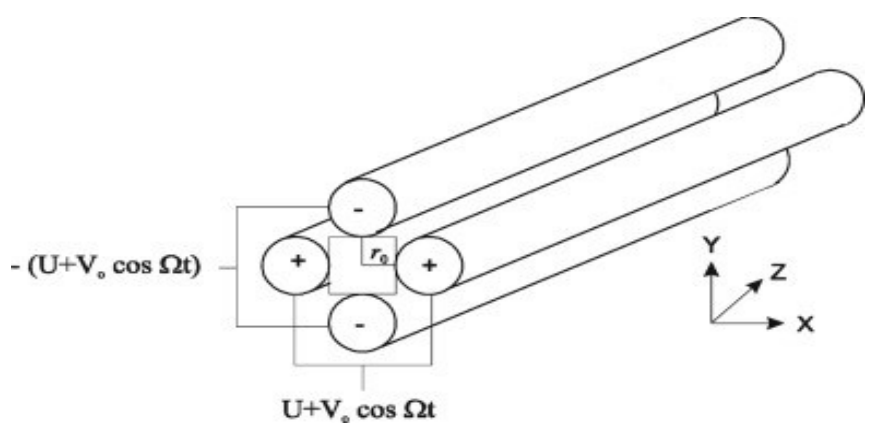

Figure 4.10: Diagram of Quadrupole mass filter. The ions enter and travel in the z-direction, while oscillating in the $\mathrm{x}-\mathrm{y}$ plane[83].

\subsubsection{Theory of operation}

The lighter ions entering the quadrupole would oscillate in phase with the potential of RF whose oscillation amplitude is increases unless they get ejected from the array or the ions 
themselves get lost by the contact with the array. However, the heavier ions would be moving through the center of quadrupole because of these heavy sizes and reach the detector device through the exit aperture of the filter. On the other hand, ions with mass greater than the selected mass will be deflected out of the beam by the DC fields.

The 2D quadrupole electric potential $\Phi$ is given by [84],

$$
\Phi(\mathrm{x}, \mathrm{y}, \mathrm{t})=\frac{\left(x^{2}-y^{2}\right)}{r_{0}^{2}} \Psi(t)
$$

Where $\Psi(\mathrm{t})$ is the potential applied to the electrodes, and $\mathrm{r}_{0}$ is the radius of the inscribed circle tangential to the inner surface of the rods. The minus sign between the $\mathrm{x}$ and the y directions is due to the opposite polarity of the horizontal and vertical rods.

Consider a potential $\Psi(\mathrm{t})= \pm\left(\mathrm{U}-\mathrm{V}_{0} \cos (\Omega \mathrm{t})\right)$ be applied to quadrupole with alternate electrodes where $\mathrm{U}$ is the $\mathrm{DC}$ voltage, $\mathrm{V}_{0}$ is the RF voltage, and $\Omega$ is angular frequency where $\Omega=2 \pi \mathrm{f}$, and $\mathrm{f}$ is the frequency in $\mathrm{Hz}$. The $2 \mathrm{D}$ quadrupole potential is given by,

$$
\Phi(\mathrm{x}, \mathrm{y}, \mathrm{t})=\frac{\left(x^{2}-y^{2}\right)}{r_{0}^{2}}\left(\mathrm{U}-\mathrm{V}_{0} \cos (\Omega \mathrm{t})\right)
$$

The motion of an ion in a two-dimensional multipole field result in force $\underset{F}{(\rightarrow)}$ on the ion, which is given by,

$$
\vec{F}=-e \nabla \Phi(x, y, t)
$$


Where e is the magnitude of the electronic charge and $\Phi$ is the multipole potential. In the $\mathrm{x}$ and $\mathrm{y}$ directions of the motion, the force can be written by,

$$
\begin{aligned}
& m \frac{d^{2} x}{d t^{2}}=-e \frac{\partial \Phi(x, y, t)}{\partial x} \\
& m \frac{d^{2} y}{d t^{2}}=+e \frac{\partial \Phi(x, y, t)}{\partial x}
\end{aligned}
$$

Equations (4.4) and (4.5) give the equation of motion in $\mathrm{x}$ and $\mathrm{y}$ directions respectively,

$$
\begin{aligned}
& m \frac{d^{2} x}{d t^{2}}=\frac{-2 e x}{r_{0}^{2}}\left(U-V_{0} \cos (\Omega t)\right) \\
& m \frac{d^{2} y}{d t^{2}}=\frac{+2 e y}{r_{0}^{2}}\left(U-V_{0} \cos (\Omega t)\right)
\end{aligned}
$$

Writing the equations of motion in terms of the parameters [85],

$$
\begin{aligned}
& a_{x}=-a_{y}=\frac{8 e U}{m \Omega^{2} r_{0}^{2}} \\
& q_{x}=-q_{y}=\frac{4 e V_{0}}{m \Omega^{2} r_{0}^{2}}
\end{aligned}
$$

and defining the variable $\xi=\frac{\Omega t}{2}$, we obtain the so-called Mathieu equations

$$
\begin{aligned}
& \frac{d^{2} x}{d \xi^{2}}+\left(a_{x}-2 q_{x} \cos 2 \xi\right) x=0 \\
& \frac{d^{2} y}{d \xi^{2}}+\left(a_{y}-2 q_{y} \cos 2 \xi\right) y=0
\end{aligned}
$$


The solution of above Mathieu equations can be calculated in the $\mathrm{x}$ and $\mathrm{y}$ direction of ion stability trajectories since the characteristic fundamental frequencies of ion motion, and its confinements are in $\mathrm{x}$ and $\mathrm{y}$-direction. When the parameters of $\mathrm{a}, \mathrm{q}$ from the Mathieu equation lies bounded region of characteristic curves $a_{0}$ and $b_{1}$ given in equation (4.12), and (4.13), one can obtain the stable and bound solutions of Mathieu equations in $\mathrm{x}$-direction[86].

$$
\begin{gathered}
a_{0}=-\frac{q^{2}}{2}+\frac{7 q^{4}}{128}+\frac{29 q^{6}}{2304}+\cdots \\
b_{1}=1-q-\frac{q^{2}}{8}+\frac{q^{3}}{64}+\frac{q^{4}}{1536}+\cdots
\end{gathered}
$$

There are mainly two modes of operation of quadrupole analyzers named RF and DC mode or RF-only mode. In the case of RF only mode of operation for quadrupole rod array, all the ions having some mass to charge ratio more than a fixed number got transmitted through the quadrupole device. Conversely, for the other mode of operation, when quadrupole is operated with $\mathrm{RF}$ and $\mathrm{DC}$ mode, the ions with small range of mass to charge ratio are allowed to maintain the stable paths and hence to reach the detector applying mass selection to the ions injected. The passage of more numbers of particles with mass to charge ratio through the filter and detector requires the constant variation of DC and RF voltage such that two filters with low mass and high mass filtration would not overlap [31]. 


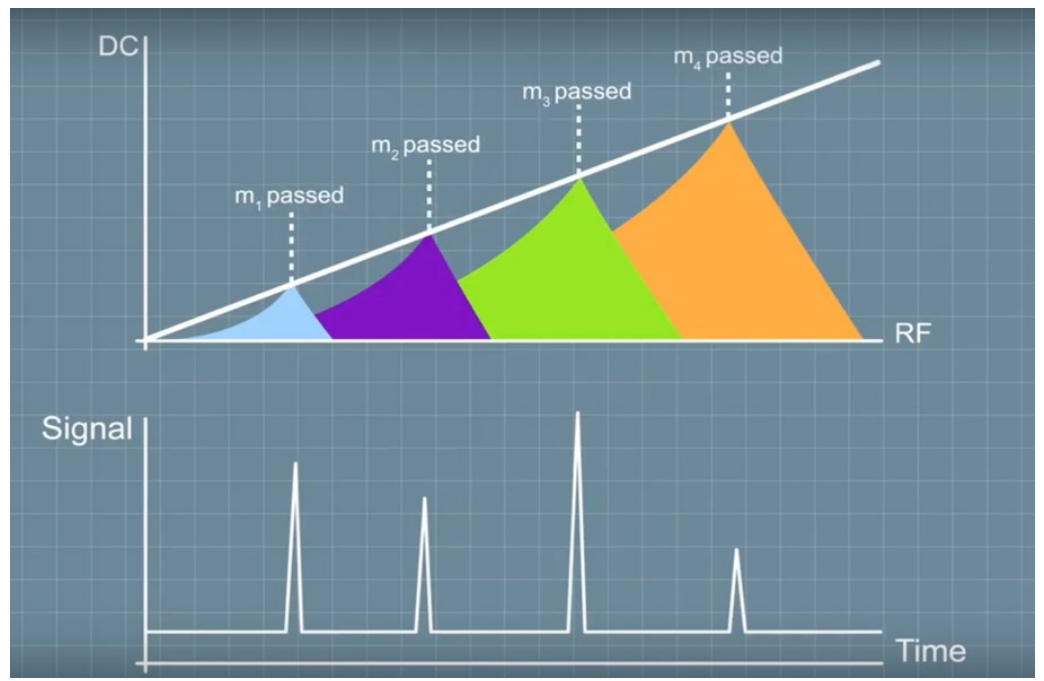

Figure 4.11: Stability diagram for a quadrupole mass filter.

\subsubsection{Channeltron Electron Multiplier}

Channeltron Electron Multiplier or a particle multiplier is the other important part of the Quadruple mass spectrometer, which is discussed in the thesis. A particle multiplier converts an ion, electron or photon entering into it to a pulse of charges at the output. This instrument plays the role of the amplifier for generating a pulse of charge at the output (which is anode here) from the particles entering through the input dynode. There are many different particle multipliers used now for mass spectrometry. For example, multi-stage CuBe types, continuous glass types and Conversion Dynode Electron Multipliers. 


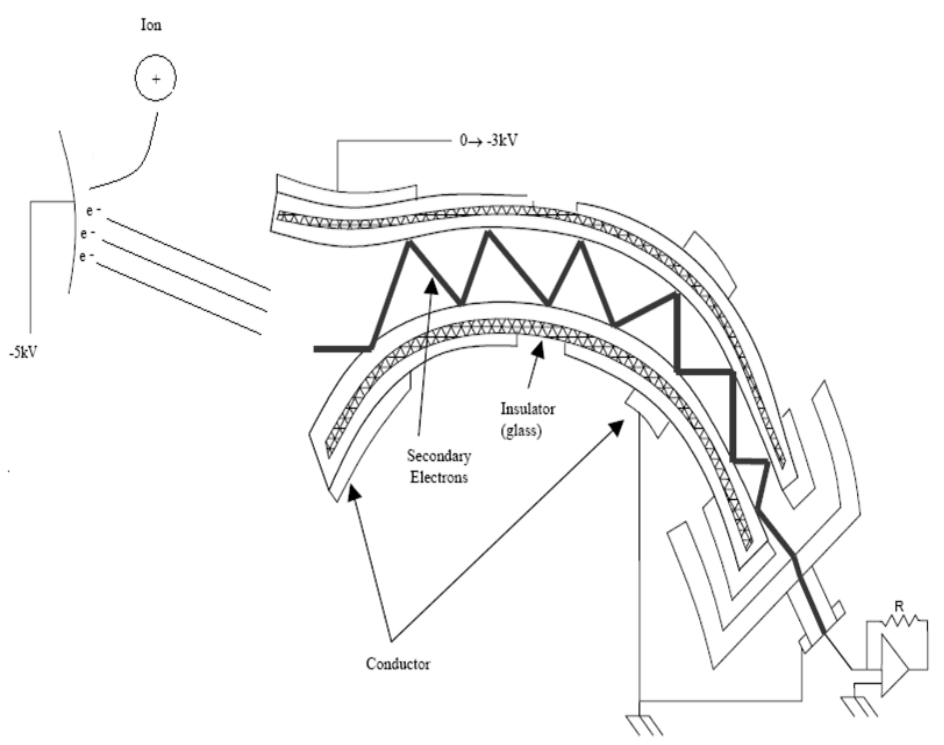

Figure 4.12: Diagram of the multiplier-dynode configuration for positive ion counting. It is adapted from [47].

The internal setup of the channel multiplier for positive ion detection is shown in Figure (4.12). In this configuration, when secondary electrons are produced, every positive ion strikes the dynodes. The dynodes are kept at a voltage $(-5 \mathrm{kV})$ for charge multiplication. The secondary electrons are then moving in the direction of the funnel-shaped inlet of the multiplier, which is kept at a voltage of $-2 \mathrm{kV}$. The signal is collected at the final stage through an avalanche process. For every electron that hits the surface of the tube, the applied electric field will induce an avalanche effect along the path, which causes more electrons to drift toward the other end of the tube. Electrons reaching the anode produce a signal which is amplified further electronically. The polarity of the multiplayer's voltage can be used to determine the polarity of ions detected. For example, in the case of negative ions to be detected, the positive input bias is needed and vice versa [31]. 
The output from the electron multiplier is commonly measured in the form of current, which is recorded through the electrometer amplifiers. However, the individual pulses can also be counted at the output. Each of the pulses is produced when an incident ion strikes the dynode and hence produces about $10^{7}$ electrons, which forms a readable pulse at the detector. The response time of the electron multiplier can be made very fast, which can result in the detection of $10^{8} \mathrm{~s}^{-1}$ ions per second count rate. The background pulses can set up the limits on the detection rates, which is usually less than one event per second signifies the improvement in the analogue techniques over the order of several magnitudes. Although the electron multiplier has a very important contribution in the quadrupole mass spectrometer, there are some disadvantages associated with it. The main drawback is that the gain of the electron multipliers decreases with time, which should be taken care of often [87]. Ion sources also degrade with time, and these changes are corrected by the design of the experiment.

\subsubsection{Merlin Automation Data System Software}

The final data measurements were recorded with the Merlin Software. This software package is a powerful analysis system that works on the Extrel's Research Command System and Extrel's MAX300-LG ${ }^{\mathrm{TM}}$ Laboratory Gas Analyzer. The special features of this software make it ideal for analyzing the applications. There are many functions performed by this software. It controls the different components of the spectrometer using the digital and analog inputs. The software provides spectral displaces for monitoring the progress of the measurement and output the mass spectra for analysis. 


\section{Chapter 5: Experimental Procedure}

\subsection{The Measurement}

The outline of the main experiment is displayed in Figure (4.2). The experiment consists of a cryogenic still with reflux column with a height $1.8 \mathrm{~m}$ and an inner diameter of $25 \mathrm{~mm}$. Multilayer insulation was used to wrap the inner column, which is then placed in a vacuum jacket. PROPAK 0.16" made of electropolished stainless steel was filled in the column in a random packing fashion. To keep the top head cooled, the Oxford Cryogenics cold refrigeration was the system used, which was operated at a fixed cooling rate. A heater was used in the re-boiler to maintain controlled fixed pressure in the vapour phase. To record the pressure, an MKS Baratron was used with an accuracy of $0.12 \%$. The reading was stable up to $0.1 \%$ throughout the runs. To keep the re-boiler heated, the heat was supplied to the re-boiler in the pair of side arms so that the liquid volume will mix properly. A differential pressure sensor attached between the top and the bottom of the boiler, which was used to measure the total volume of the liquid re-boiler about $300 \mathrm{cc}$. The electrical power supply to the boiler gave the vapour flow rate in the column, and the external heat contribution to the boiling was about $2 \%$. The volume flow was basically $0.25 \mathrm{~g} / \mathrm{s}$ for the krypton, and other gases had similar molar flows. For any packed column there is a characteristic vapour or liquid flow rate known as the flooding flow, above which liquid holdup in the column rises sharply along with the pressure drop. From the PROPAK data sheet [82], the flooding flow vapour velocity for a column such as ours is about $1 \mathrm{~m} / \mathrm{s}$. As the flows in our measurements were of order $0.05 \mathrm{~m} / \mathrm{s}$ the operation is safely away from the flooding limit. From these measurements it is concluded that the still was operated in a 
mode in which we could regard the pressure and temperature constant and the conditions stable. The relative abundance of isotopes either at the top or the bottom of the column was measured with the mass spectrometer. The capillary tubes of $0.5 \mathrm{~mm}$ ID were used for sampling, and the procedure of purging the capillaries before taking each measurement was followed. The mass spectrometer used for the measurement was made of an external quadrupole with a $19 \mathrm{~mm}$ bore and a 1:2000 mass resolution. A channel multiplier was used to detects the ions, and to prevent the non-linearity due to pile up seen through the pulse counting electronics, a current preamplifier was used along with the other apparatus discussed above. During each measurement, the current detected was sampled while keeping the fixed quadrupole scanned mass range. The time for each scan was approximately 1 second, and the mass spectrum was made from the averaged few thousands of scans. After this measurement, the new reading was recorded from the opposite end of the column. This procedure was repeated for several days while recording approximately ten readings per day. For each of the three gasses, the typical spectra from one of such measurements are shown in Figures (5.1), (5.3) and (5.5). From the figures, one can see that the isotopic peaks are well resolved, and even the isotopes with the least abundance can be clearly seen. The other thing shown in this figure is the mass range of the different samples for each of the isotopes. A broad mass range spectrum for each of the gas was recorded. For example, for the argon gas data, smaller peaks can be seen at the mass of about $32\left(\mathrm{O}_{2}\right)$ and $44\left(\mathrm{CO}_{2}\right)$. No other background peaks were recorded at the other higher masses for any of the gases used in the measurement. Therefore, the presence of other contaminants was small for any measurement. 
For the observation of the VPIE for the xenon, which would be approximately lass than $1 \%$ in the full column, it is critical to take care of the systematic errors. This is widely accomplished with the design of the experiment. The intensities of isotopes were recorded relative to the total number for all the isotopes of the given fluid. This will help out to correct any present time variation measurement of the mass spectrometer system. The changes in the relative intensities' measurements from the top and the bottom of the spectrometer gave the isotopic separations from the whole measurements. Hence, the mass dependence or the rate dependence of the spectrometer can be eliminated. For the extraction of the VPIE for xenon from the distillation separation data, the calibration of the still and full understanding of the mass transfer properties was needed. It was done by taking initial measurements with argon and krypton for which VPIE was already known and then to figuring out these results, which is a useful method to extrapolate to xenon. The first recorded data signifies the best method to assume the constant reading of HETP (Height of the Equivalent Theoretical Plate). This was decided before the xenon data was taken, and therefore the following analysis can be done blindly.

\subsection{Data analysis}

From the mass spectrum plots, we can measure the enrichment from the top to the bottom of the distillery, the number of theoretical plates, and the Height of the equivalent Theoretical Plate (HETP). The isotopes are seen to be cleanly separated and sit on a small background. Various methods for extracting the isotopic abundances are being explored. For the first simple extraction, a linear background is assumed under each peak. This is determined by a simple fit and the residue to taken to be a measure of the abundance. The peaks 
are not Gaussian or any other simple form. A second analysis was done in which a single experimental shape was taken from one of the strong peaks and this was used to fit the rest of the peaks in the spectra.

In addition, a slow decline in the overall rate was observed over the experiments which spanned several days. To correct for this, the strongest line in the spectrum was used to normalize all other peaks in the spectrum. Thus, we used ${ }^{40} \mathrm{Ar}$ for the argon runs, ${ }^{84} \mathrm{Kr}$ for the krypton runs and ${ }^{132} \mathrm{Xe}$ for the xenon runs. The relative abundance of each peak, for example ${ }^{136} \mathrm{Xe}$, was normalized with respect to the main peak as:

$$
\text { normalized with main peak }=\frac{A^{136} \mathrm{Xe}}{A^{132} \mathrm{Xe}}
$$

We average all of the top and bottom normalized amplitudes of a single peak separately, and then calculate the percent change in abundance as:

$$
\text { Enrichment }=\frac{\left(\left({ }^{136} \mathrm{Xe} /{ }^{132} \mathrm{Xe}\right)_{\text {top }}-\left({ }^{136} \mathrm{Xe} /{ }^{132} \mathrm{X}\right)_{\mathrm{bottom}}\right)}{\left(\left({ }^{136} \mathrm{Xe} /{ }^{132} \mathrm{X}\right)_{\mathrm{top}}+\left({ }^{136} \mathrm{Xe} /{ }^{132} \mathrm{X}\right)_{\mathrm{bottom}}\right)} \times 200 \%
$$

We calculate the enrichment between corresponding individual pairs of top/bottom measurements, and then calculated the average of enrichment measurements as:

$$
\bar{A}_{\text {enrichment }}=\frac{1}{n} \sum_{i=1}^{n} A_{i}
$$

The standard deviation of the amplitude $\left(\mathrm{s} \sigma_{A}\right)$ is:

$$
\sigma_{A}=\sqrt{\frac{\sum_{i=1}^{n}\left(A_{i}-\bar{A}\right)^{2}}{n-1}}
$$


The uncertainty of the slope measurement was found using error propagation as:

$$
\sigma_{\text {slope }}=S D / \sqrt{N-1}
$$

The number of theoretical plates, $\mathrm{N}$, was found by:

$$
N=\frac{\Delta E}{\delta p / p}
$$

where $\Delta \mathrm{E}$ is the change in enrichment within a range of isotopes and $\delta \rho / \rho$ is the vapour pressure difference of the same range.

The HETP for the distillery was then calculated as:

$$
H E T P=h / N
$$

Where $\mathrm{h}$ is the total height of the distillery column ( $6 \mathrm{ft}$ or $\sim 1.83 \mathrm{~m}$ ).

The height of the distillery necessary to be built can be calculated by:

$$
h=H E T P .\left(\frac{\Delta E}{\delta p / p}\right)
$$

\subsubsection{Corrections to the still analysis}

In order to extract the physically interesting quantities such as the VPIE from the slopes of the enrichment factors it is necessary to account in some way for the still properties. It is argued that the basic still parameter - the HETP is the same for the three noble fluids when the still is used for inert fluids, under the same conditions of pressure and vapour flow, and at the very low flooding conditions used in these measurements. The reported value of the VPIE for argon is then used to calibrate this HETP. The krypton data are used to test the 
extrapolation from argon to krypton and get an estimate of the possible systematic error. Finally, the VPIE for xenon is determined using the common HETP.

Following Wagner [88] for conditions of 100\% reflux, the height of a vapour transfer unit is the same as the HETP and can be written

$$
H E T P=\left(\frac{\pi(\varepsilon-h) u_{V}}{4 D_{V}}\right)^{0.5}\left[1+\left(\frac{h D_{V} M_{L} \rho_{V}}{(\varepsilon-h) D_{L} M_{V} \rho_{L}}\right)^{0.5}\right] \frac{\chi^{0.5}}{a_{e}}
$$

Where:

$\varepsilon$ is the packing void fraction

$\mathrm{h}$ is the liquid volume in the packing

$\mathrm{u}_{\mathrm{V}}$ is the vapour velocity through the packing

$D_{V}$ is the vapour phase self-diffusion coefficient

$\mathrm{D}_{\mathrm{L}}$ is the liquid phase self-diffusion coefficient

$\mathrm{M}_{\mathrm{L}}$ and $\mathrm{M}_{\mathrm{V}}$ are the liquid and vapour molecular masses (equal in this case)

$\rho_{\mathrm{L}}$ and $\rho_{\mathrm{V}}$ are the liquid and vapour densities

$\chi$ is an effective height that corrects for turbulence

$\mathrm{a}_{\mathrm{e}}$ is an effective area of packing that corrects for the whetted area

We expect the liquid holdup to be a small fraction of the void volume in the packing and thus expect the leading term to dominate. The vapour phase diffusion constants at the boiling point are very similar for these noble materials. The still was operated at a constant value of the vapour velocity and we do not expect the packing specific parameters to vary with the noble fluid. Thus, the overall values of HETP are taken to be the same. The measurements were made at the respective boiling points of the substances, but the literature quotes VPIE values at the triple point. Following Lopes, we assume that the VPIE for each 
substance varies as $1 / T^{* 2}$ where $T^{*}$ is an effective temperature equal to the Kelvin temperature divided by the depth of the Lennard-Jones potential. Thus, for the correction needed the VPIE is scaled by $1 / T^{2}$.

\subsection{Taking Data}

To start a measurement from one of the sample lines either the top or the bottom of the distillation column, the sample valves are first closed, and the manifold region is pumped out. The pump is then valving off and the chosen sample valve is opened. After a short time, the sample valve is closed, and the manifold is again pumped out. The process is repeated one further time and then the sample valve is left open. This process is designed to ensure that gas at the input to the spectrometer is representative of the required sample location. Once the spectrometer pressure has recovered to the normal operating point $(6 \mathrm{x}$ $10^{-6} \mathrm{mb}$ ) scans of the spectrometer are commenced. A single measurement is based on summing 1000 scans typically taking 20 minutes. The measurements reported here are based on several runs alternating between the sample lines at the top and bottom of the packing. Data was taken in four-run periods as listed in Table (5.1).

\begin{tabular}{|c|c|c|c|}
\hline Run & Date & Number of runs & Target element \\
\hline 1 & October 5-12,2018 & 49 runs. & Argon \\
\hline 2 & November 6-7,2018 & 17 runs & Krypton \\
\hline 3 & April 11-19,2019 & 187 runs. & Krypton \\
\hline 4 & April 23-26,2019 & 200 runs & Xenon \\
\hline
\end{tabular}

Table 5.1: Data taking runs. 
- Ar Mass spectrum and result

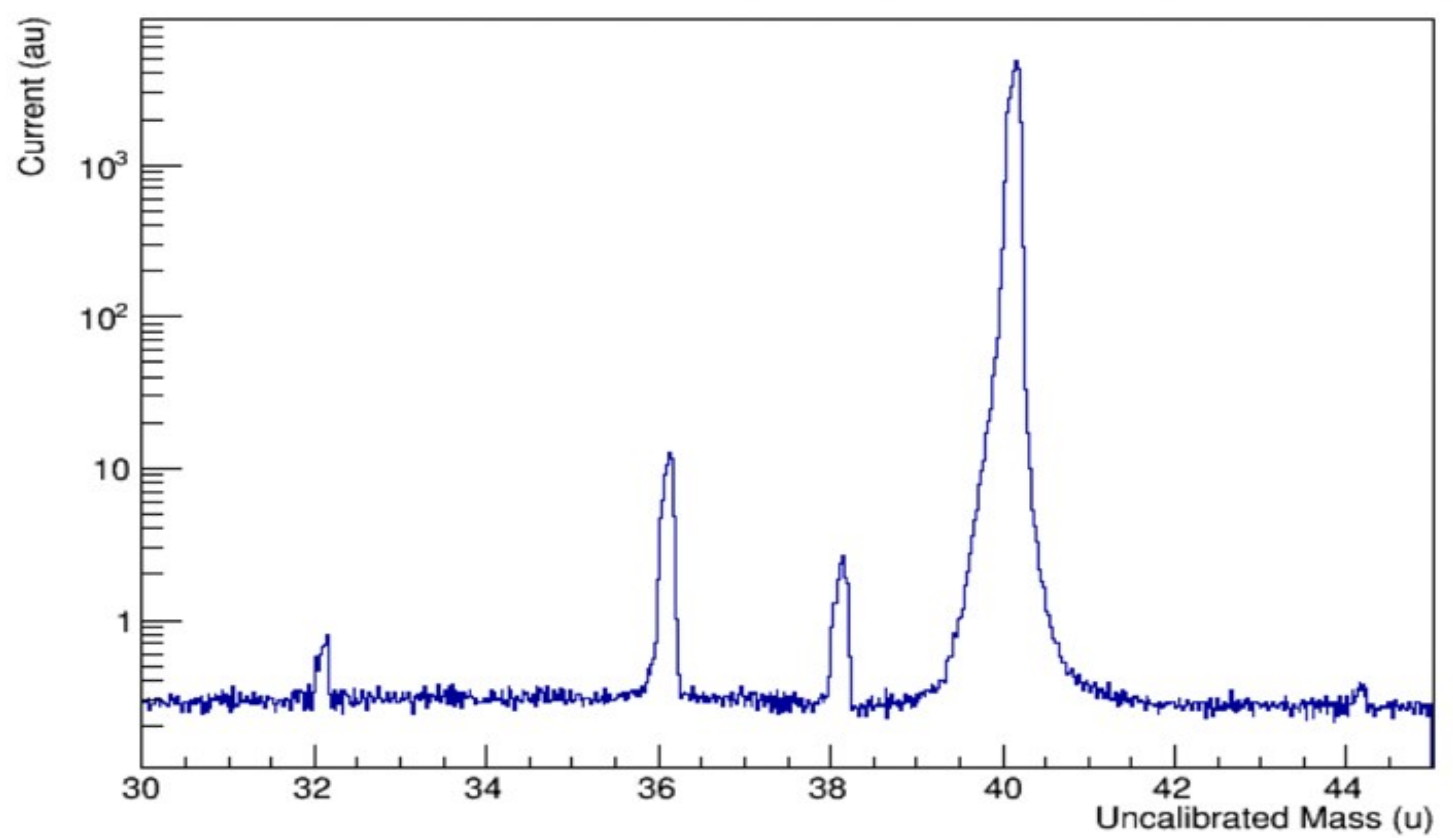

Figure 5.1: Measured spectrum for Ar. The mass peaks from 36 to 40 are clearly seen. 


\begin{tabular}{|c|c|c|c|}
\hline \multirow{2}{*}{ Trial -2018 } & \multicolumn{2}{|c|}{ Fractional Difference Top-Bottom } & \multirow[t]{2}{*}{ slope } \\
\hline & ${ }^{36} \mathrm{Ar}$ & ${ }^{38} \mathrm{Ar}$ & \\
\hline Oct-5- T-B & 2.96 & 3.51 & 0.27 \\
\hline Oct-5- T-B & 5.26 & 2.73 & -1.27 \\
\hline Oct-5- T-B & 8.71 & 2.95 & -2.88 \\
\hline Oct-5- T-B & 13.19 & 5.08 & -4.06 \\
\hline Oct-5- T-B & 11.93 & 4.10 & -3.92 \\
\hline Oct-5- T-B & 19.16 & 7.54 & -5.81 \\
\hline Oct-5- T-B & 18.82 & 9.21 & -4.80 \\
\hline Oct-6- T-B & 22.21 & 12.99 & -4.61 \\
\hline Oct-6- T-B & 27.44 & 8.80 & 9.32 \\
\hline Oct-6- T-B & 23.95 & 15.77 & -4.09 \\
\hline Oct-6- T-B & 24.56 & 10.11 & -7.22 \\
\hline Oct-6- T-B & 24.60 & 5.96 & -9.32 \\
\hline Oct-6- T-B & 25.96 & 8.19 & -8.89 \\
\hline Oct-7- T-B & 21.80 & 11.31 & -5.24 \\
\hline Oct-7- T-B & 26.37 & 8.55 & -8.91 \\
\hline Oct-7- T-B & 24.08 & 21.88 & -1.10 \\
\hline Oct-9- T-B & 26.57 & 11.10 & -7.74 \\
\hline Oct-9- T-B & 26.25 & 11.84 & -7.20 \\
\hline Oct-10- T-B & 28.78 & 17.17 & -5.80 \\
\hline Oct-10- T-B & 24.49 & 12.21 & -6.14 \\
\hline Oct-11-T-B & 26.14 & 13.03 & -6.56 \\
\hline Oct-11-T-B & 26.74 & 9.73 & -8.51 \\
\hline Oct-12-T-B & 20.81 & 5.51 & -7.65 \\
\hline Oct-12-T-B & 22.27 & 4.73 & -8.81 \\
\hline Average Enrichment\% & 24.88 & 11.11 & -6.30 \\
\hline Std. Dev. & 2.19 & 4.38 & 2.28 \\
\hline Error & 0.49 & 0.95 & 0.50 \\
\hline
\end{tabular}

Table 5.2: Ar calculating the enrichment between top and bottom measurements, and calculating the slope for every enrichment measurement. Runs from the first day. Oct 5 show results prior to the column reaching equilibrium. Analysis of the equilibrium enrichment start with the Oct 6 data. 


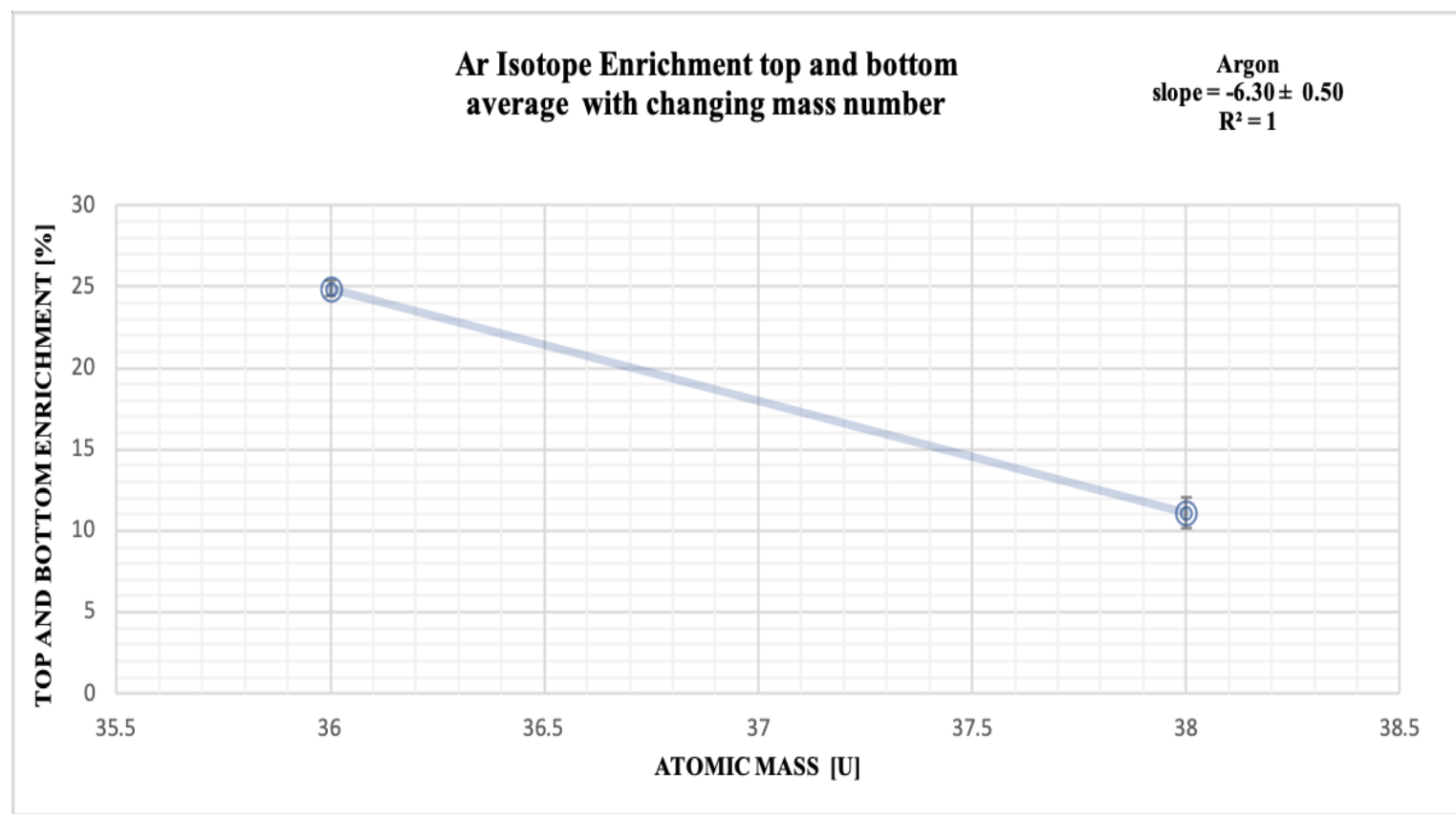

Figure 5.2: Measured argon enrichment percentage as a function of mass. The slope is $-6.30 \%$ /Mass Unit. For this analysis the ${ }^{40} \mathrm{Ar}$ peak has been used to normalize the runs. 
- Kr Mass spectrum and result

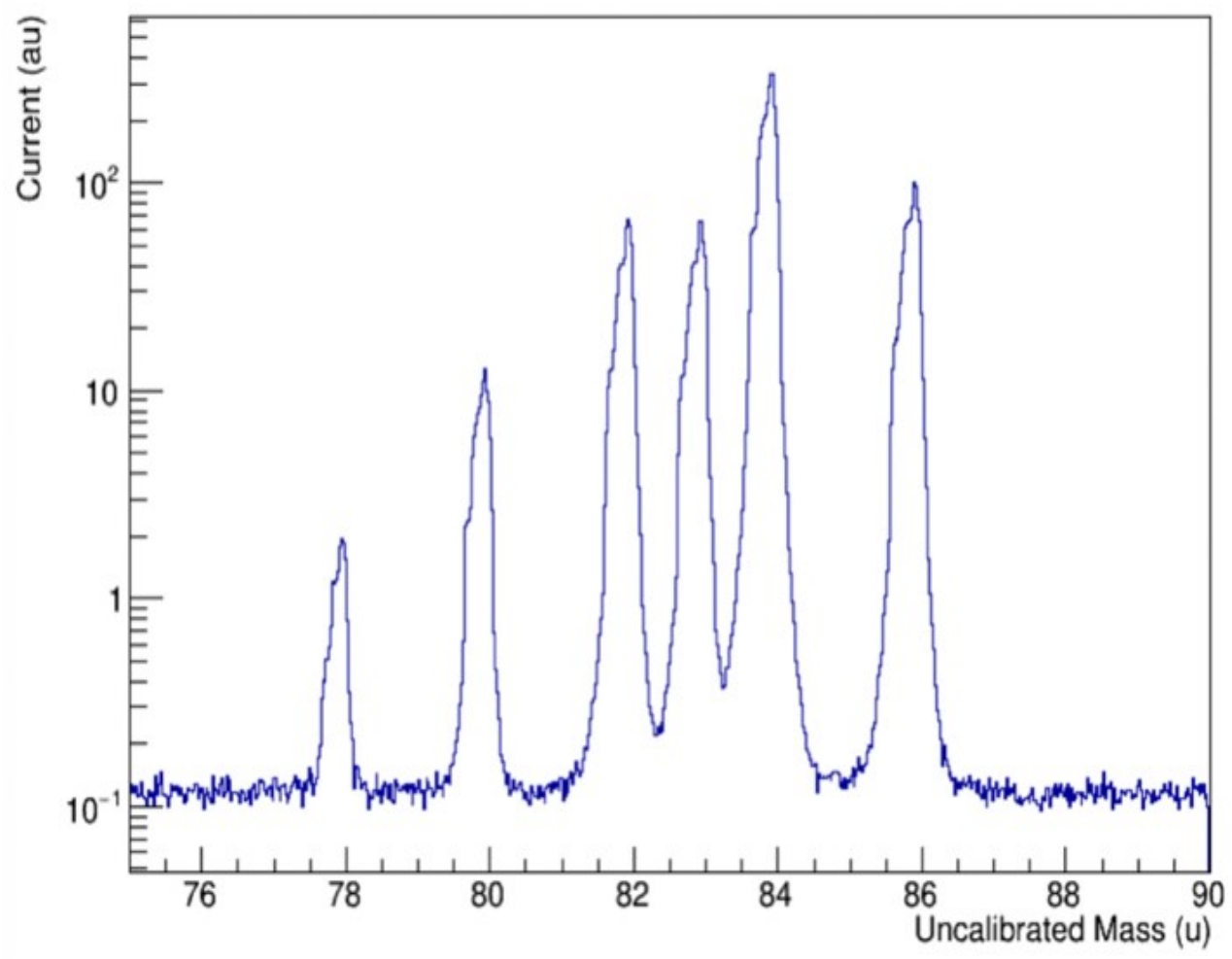

Figure 5.3: Mass spectrum for krypton. The mass peaks from 78 to 86 are clearly seen. 


\begin{tabular}{|c|c|c|c|c|c|c|}
\hline \multirow{2}{*}{ Trial-2019 } & \multicolumn{5}{|c|}{ Fractional Difference Top-Bottom } & \multirow{2}{*}{ slope } \\
\cline { 2 - 5 } & ${ }^{78} \mathrm{Kr}$ & ${ }^{80} \mathrm{Kr}$ & ${ }^{82} \mathrm{Kr}$ & ${ }^{83} \mathrm{Kr}$ & ${ }^{86} \mathrm{Kr}$ & \\
\hline Apr-17 & 1.09 & 0.53 & 0.46 & 0.28 & -0.56 & -0.19 \\
\hline Apr-17 & 3.58 & 1.82 & 0.87 & 0.15 & -1.05 & 0.57 \\
\hline Apr-17 & 4.45 & 2.61 & 1.06 & 0.49 & -1.12 & -0.69 \\
\hline Apr-18 & 5.76 & 2.93 & 0.98 & 0.34 & -1.72 & -0.92 \\
\hline Apr-18 & 7.01 & 3.65 & 1.56 & 0.82 & -1.70 & -1.06 \\
\hline Apr-18 & 6.70 & 3.97 & 2.00 & 0.72 & -1.93 & -1.07 \\
\hline Apr-18 & 5.10 & 2.84 & 1.30 & 0.83 & -2.03 & -0.96 \\
\hline Apr-18 & 7.57 & 3.91 & 1.77 & 0.77 & -1.97 & -1.16 \\
\hline Apr-18 & 6.96 & 4.31 & 1.70 & 1.03 & -1.48 & -1.06 \\
\hline Apr-18 & 4.24 & 2.45 & 1.39 & 0.62 & -1.37 & -0.69 \\
\hline Apr-19 & 8.25 & 4.19 & 1.93 & 0.58 & -1.77 & -1.24 \\
\hline Apr-19 & 4.84 & 3.17 & 1.62 & 0.78 & -1.57 & -0.80 \\
\hline Apr-19 & 7.53 & 3.02 & 1.49 & 0.77 & -1.46 & -1.059 \\
\hline Apr-19 & 5.28 & 3.01 & 1.31 & 0.86 & -1.63 & -0.85 \\
\hline Apr-19 & 6.45 & 3.70 & 1.61 & 0.75 & -1.67 & -0.98 \\
\hline Average Enrichment\% & 6.44 & 3.47 & 1.61 & 0.75 & -1.67 & -0.94 \\
\hline Std. Dev. & 1.24 & 0.61 & 0.23 & 0.15 & 0.23 & 0.19 \\
\hline Error & 0.34 & 0.17 & 0.06 & 0.04 & 0.06 & 0.05 \\
\hline
\end{tabular}

Table 5.3: Kr calculating the enrichment between top and bottom measurements and calculating the slope for every enrichment measurement. 


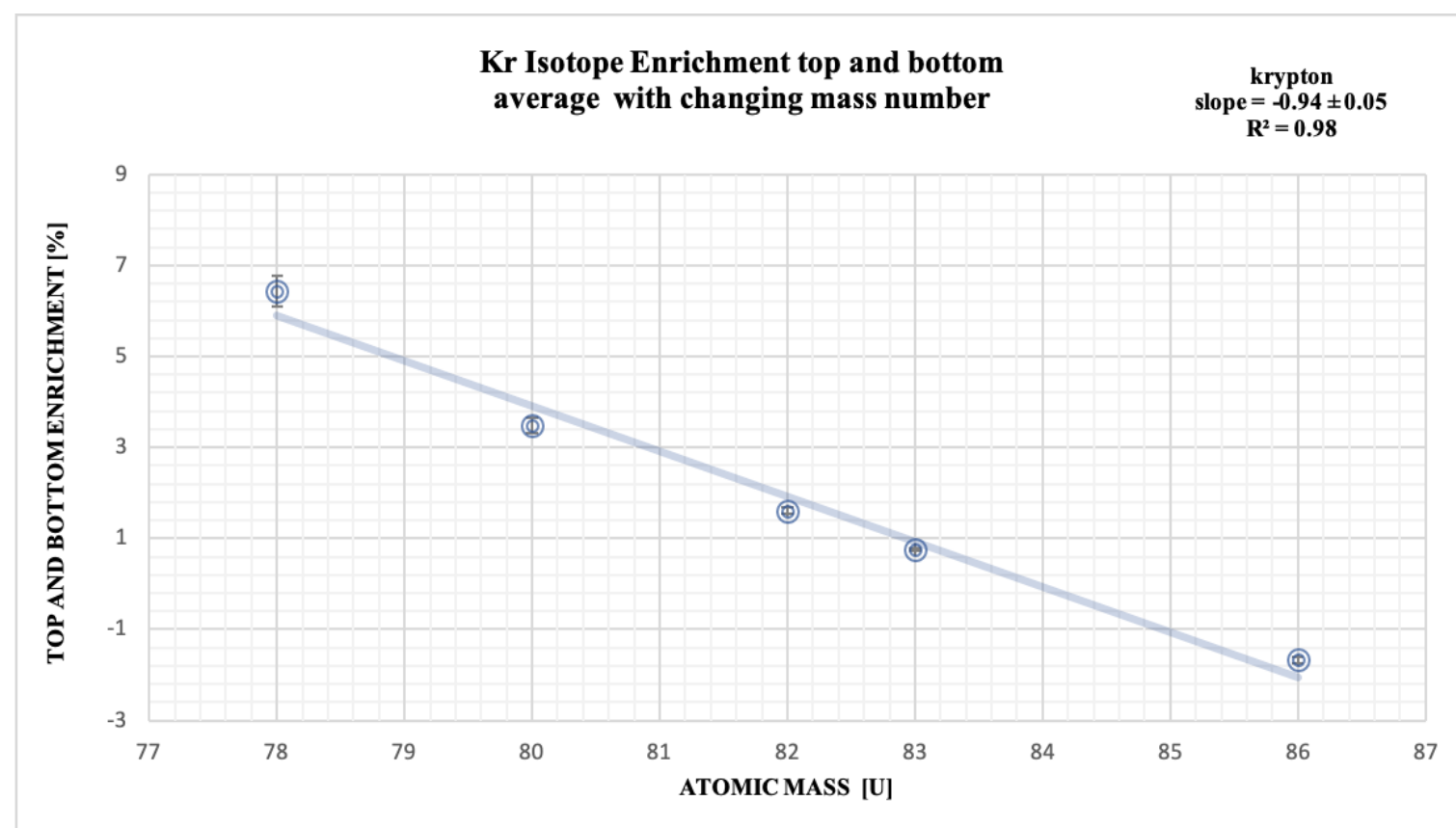

Figure 5.4: Measured krypton enrichment percentage as a function of mass. The slope is $-0.94 \%$ /Mass Unit. For this analysis the ${ }^{84} \mathrm{Kr}$ peak has been used to normalize the runs. 
- Xe Mass spectrum and result

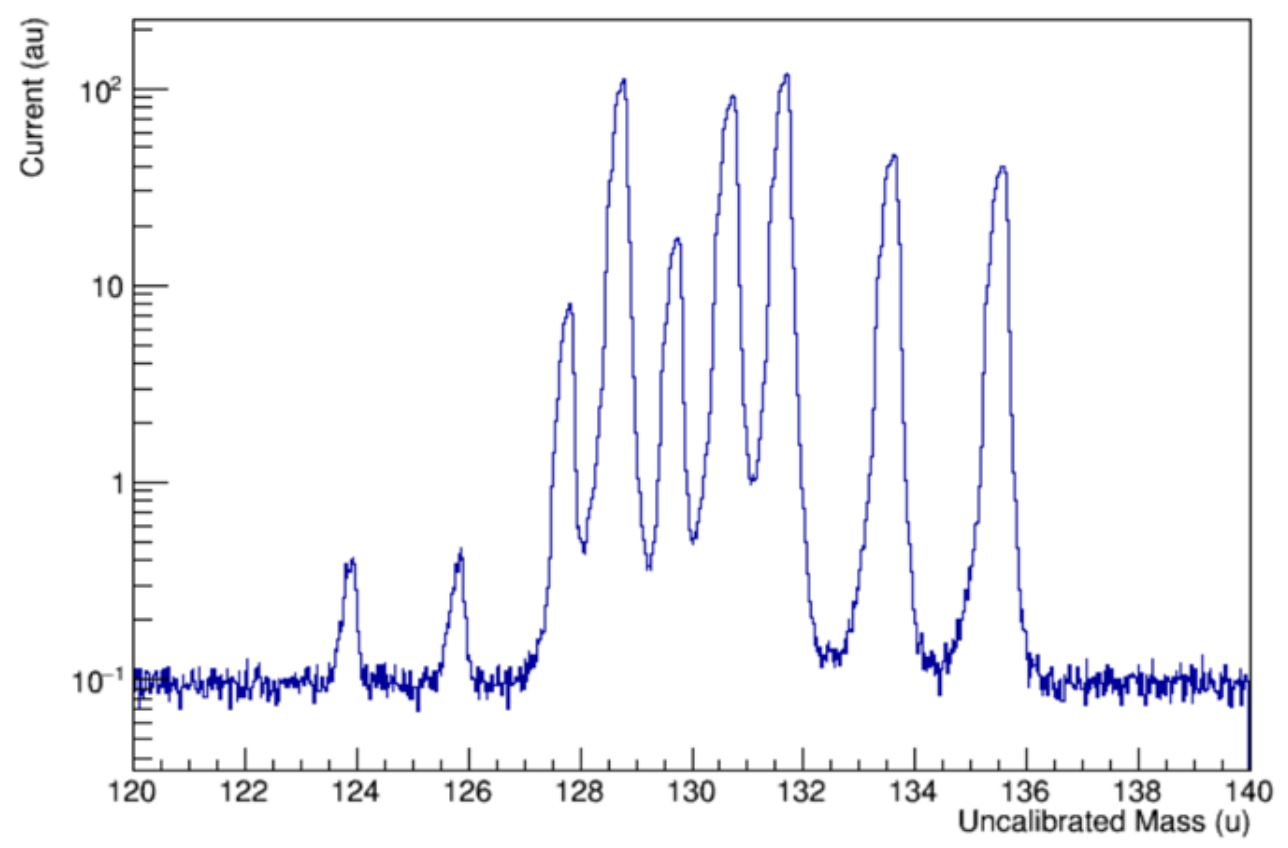

Figure 5.5: Mass spectrum for xenon. The mass peaks from 124 to 136 are clearly seen. 


\begin{tabular}{|c|c|c|c|c|c|c|c|}
\hline \multirow{2}{*}{ Trial-2019 } & \multicolumn{6}{|c|}{ Fractional Difference Top-Bottom } & \multirow{2}{*}{ slope } \\
\hline & ${ }^{128} \mathrm{Xe}$ & ${ }^{129} \mathrm{Xe}$ & ${ }^{130} \mathrm{Xe}$ & ${ }^{131} \mathrm{Xe}$ & ${ }^{134} \mathrm{Xe}$ & ${ }^{136} \mathrm{Xe}$ & \\
\hline Apr-23 & -0.24 & -0.14 & -0.37 & -0.24 & -0.26 & -0.35 & -0.23 \\
\hline Apr-23- & -0.29 & -0.15 & -0.45 & 0.07 & 0.22 & 0.20 & -0.07 \\
\hline Apr-23- & 1.79 & 0.56 & 1.35 & 0.24 & -0.29 & 0.56 & -0.15 \\
\hline Apr-24 & 1.18 & 0.49 & 0.64 & 0.31 & -0.49 & -0.68 & -0.33 \\
\hline Apr-24 & 1.03 & 0.49 & 0.51 & 0.29 & -0.29 & -0.82 & -0.29 \\
\hline Apr-24 & 0.56 & -0.01 & 0.23 & -0.01 & -0.50 & -0.62 & -0.31 \\
\hline Apr-25 & -0.65 & 0.56 & -0.24 & 0.12 & -0.40 & -0.43 & -0.31 \\
\hline Apr-25 & -1.55 & 0.32 & 0.19 & 0.18 & -0.27 & -0.29 & -0.40 \\
\hline Apr-25 & 0.69 & 0.41 & 0.89 & -0.06 & -0.63 & -1.49 & -0.48 \\
\hline Apr-25 & 0.16 & 0.32 & 0.44 & 0.05 & -0.50 & -1.25 & -0.19 \\
\hline Apr-25 & 0.34 & 0.27 & 0.94 & 0.18 & -0.29 & 0.04 & -0.28 \\
\hline Apr-25 & -0.34 & 0.41 & 1.04 & 0.10 & -0.47 & -0.77 & -0.21 \\
\hline Apr-26 & -1.44 & 0.53 & 0.52 & 0.02 & -0.03 & -0.46 & -0.24 \\
\hline Apr-26 & -0.65 & 0.08 & 0.36 & 0.04 & -0.64 & -0.72 & -0.07 \\
\hline Apr-26 & 2.84 & 0.65 & 0.86 & 0.04 & -0.61 & 0.12 & -0.28 \\
\hline Apr-26 & 0.26 & 0.52 & -0.71 & 1.66 & -1.42 & -1.60 & -0.27 \\
\hline Average & -2.20 & 1.52 & -3.15 & 0.19 & -0.42 & -0.70 & -0.15 \\
\hline Std. Dev. & 11.46 & 4.66 & 15.62 & 0.39 & 8.06 & 0.47 & 0.29 \\
\hline Error & 2.70 & 1.10 & 3.68 & 0.09 & 1.90 & 0.11 & 0.07 \\
\hline
\end{tabular}

Table 5.4: Xe calculating the enrichment between top and bottom measurements and calculating the slope for every enrichment measurement.

\begin{tabular}{|c|c|c|c|c|c|c|c|}
\hline Trial & \multicolumn{6}{|c|}{ Fractional Difference Top-Bottom } & \multirow{2}{*}{ slope } \\
\hline April-26, 2019 & ${ }^{128} \mathrm{Xe}$ & ${ }^{129} \mathrm{Xe}$ & ${ }^{130} \mathrm{Xe}$ & ${ }^{131} \mathrm{Xe}$ & ${ }^{134} \mathrm{Xe}$ & ${ }^{136} \mathrm{Xe}$ & \\
\hline 5 runs $\mathrm{T}-5$ runs $\mathrm{B}$ & -0.65 & 0.08 & 0.36 & 0.04 & -0.64 & -0.72 & -0.07 \\
\hline 5 runs $T-5$ runs $B$ & 2.84 & 0.65 & 0.86 & 0.04 & -0.61 & 0.12 & -0.28 \\
\hline 5 runs $\mathrm{T}-5$ runs $\mathrm{B}$ & 0.26 & 0.52 & -0.71 & 1.66 & -1.42 & -1.60 & -0.27 \\
\hline Average & 0.81 & 0.42 & 0.17 & 0.58 & -0.89 & -0.73 & -0.21 \\
\hline Std. Dev. & 1.81 & 0.30 & 0.80 & 0.94 & 0.46 & 0.86 & 0.12 \\
\hline Error & 1.04 & 0.17 & 0.46 & 0.54 & 0.27 & 0.49 & 0.08 \\
\hline
\end{tabular}

Table 5.5: For this analysis the ${ }^{132} \mathrm{Xe}$ peak has been used to normalize the runs. Peak 124 and 126 have been removed from the analysis because the fluctuation in these small peaks is large. These runs of Xe were used to do the analysis because there was an improvement in setting for mass spectrum which gave better resolution. 


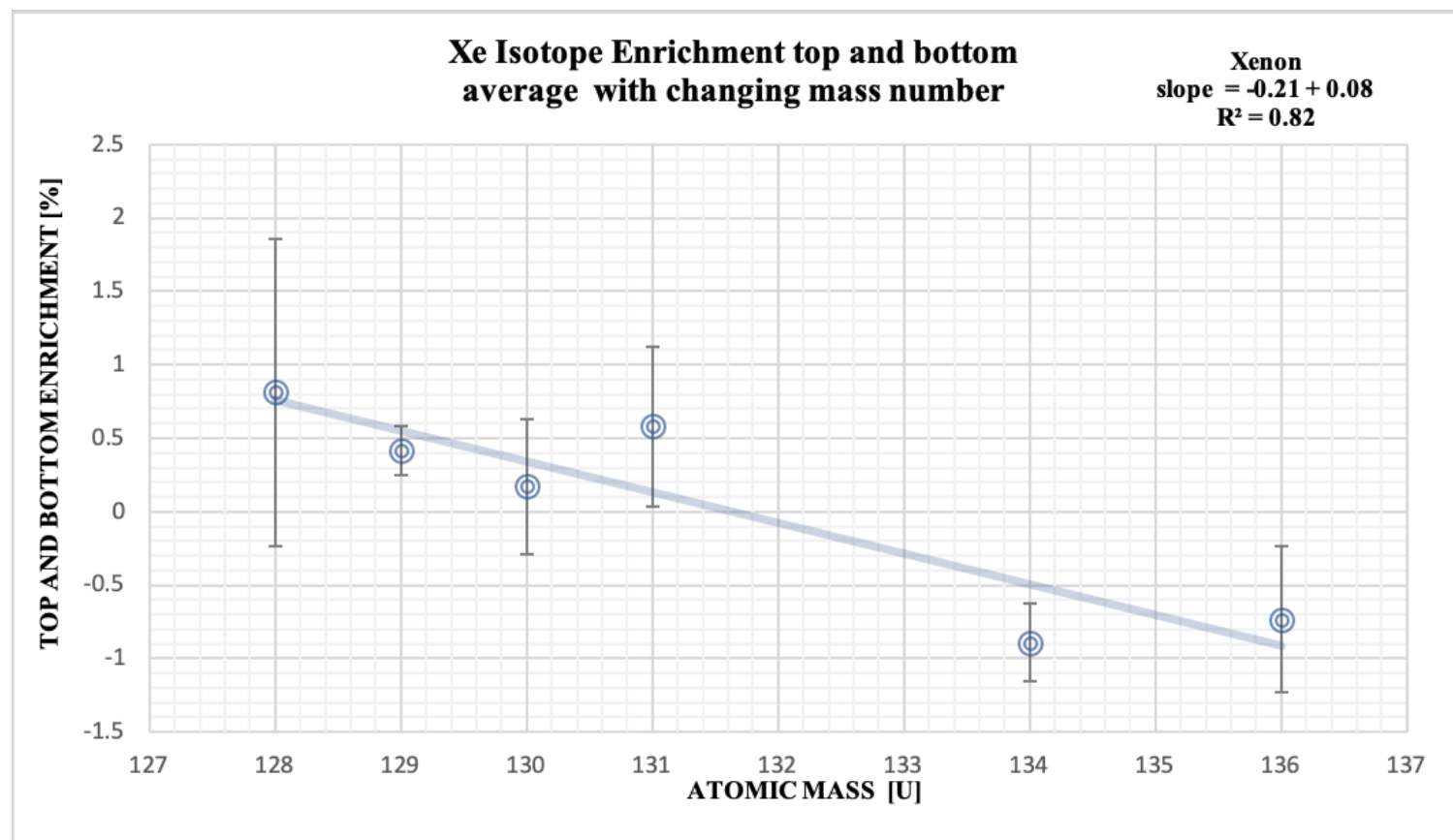

Figure 5.6: Measured xenon enrichment percentage as a function of mass. The slope is $-0.21 \%$ /Mass Unit.

For each element $(\mathrm{Ar}, \mathrm{Kr}, \mathrm{Xe})$ the slope of the line determines the separation per mass unit for the still and this is proportional to the VPIE per mass unit for each element. The linear relationship with mass is expected from theory but has not been previously reported. 


\section{- Alternative Analysis}

An alternative analysis was carried out by Caio Licciardi. First, one peak in the spectrum is chosen to give a standard peak shape. Then a maximum likelihood fit to the complete spectrum is done assuming the data to be a sum of peaks of the chosen shape plus a background. This gives the amplitude of each peak in the spectrum. This analysis gives consistent results with somewhat smaller errors especially for xenon.
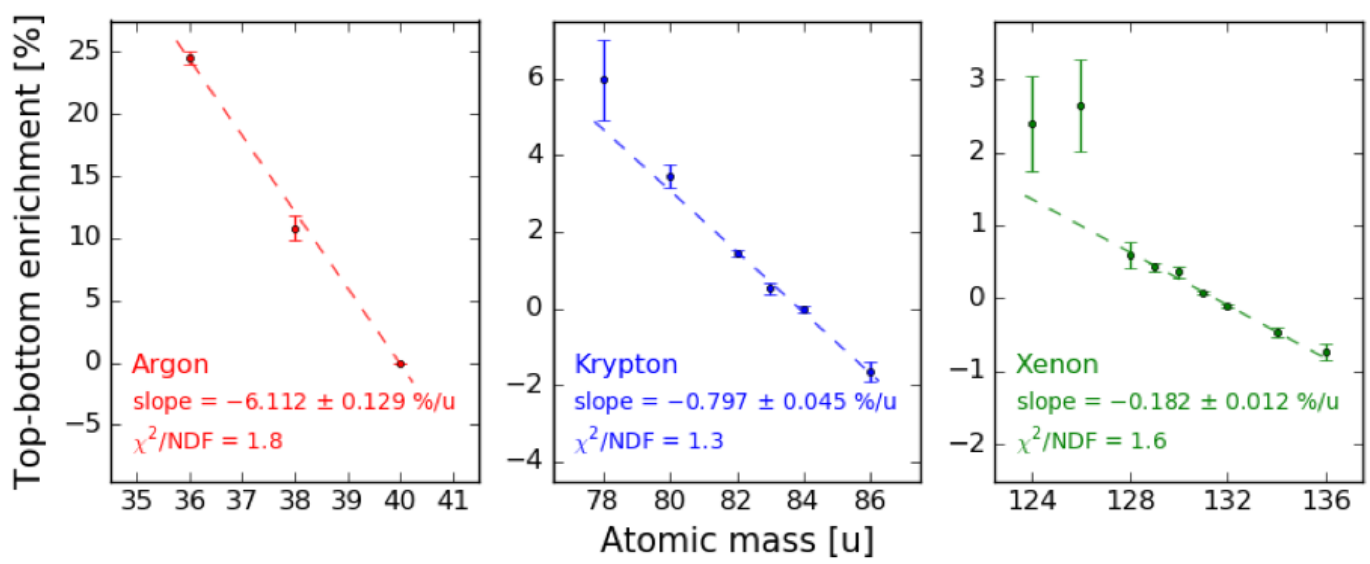

Figure 5.7: Alternative analysis for fractional difference between the top and bottom of the column for each isotope (by Caio Licciardi). 


\section{- Final result}

\begin{tabular}{|c|c|c|c|c|c|}
\hline \multirow{2}{*}{ Element } & \multicolumn{2}{|c|}{ Alamre } & \multicolumn{2}{c|}{ Licciardi } & Tew \\
\cline { 2 - 6 } & Slope & Exp. VPIE at TP & Slope & Exp. VPIE & \multirow{2}{*}{ VPIE at TP } \\
& {$[\% \mathrm{u}]$} & & $-6.11 \pm 0.13$ & $1.64 \mathrm{E}-03$ & $1.65 \mathrm{E}-03$ (Exp) \\
\hline $\mathrm{Ar}$ & $-6.30 \pm 0.50$ & $1.64 \mathrm{E}-03$ & $-0.80 \pm 0.05$ & $2.10 \mathrm{E}-04$ & $2.50 \mathrm{E}-04$ (Exp) \\
\hline $\mathrm{Kr}$ & $-0.94 \pm 0.05$ & $2.33 \mathrm{E}-04 \pm 0.12 \mathrm{E}-04$ & $-0.18 \pm 0.01$ & $4.75 \mathrm{E}-05$ & $5.17 \mathrm{E}-05$ (theory) \\
\hline $\mathrm{Xe}$ & $-0.21 \pm 0.08$ & $5.28 \mathrm{E}-05 \pm 2.0 \mathrm{E}-05$ & -0.18 & \\
\hline
\end{tabular}

Table 5.6: Measured enrichment factors for the cryogenic still together with the inferred VPIE at the triple point. Tew reports experimental data for $\mathrm{Ar}$ and $\mathrm{Kr}$ and theoretical value for Xe. Uncertainties for the VPIE measurements include only the error on the slopes obtained from the fit results. See text for further discussion. 


\subsubsection{Discussion}

For Argon, the reported VPIE given by the difference in vapour pressure of ${ }^{36} \mathrm{Ar}$ and ${ }^{40} \mathrm{Ar}$ divided by the mean vapour pressure is 0.0066 . We correct this to the boiling point and express it as the VPIE per mass unit to get 0.00152. From the slope of the argon enrichment curve we determine the number of theoretical plates is 41.4 and thus HETP is $4.4 \mathrm{~cm}$. This compares with the manufacturer's values of about $3 \mathrm{~cm}$ for hydrocarbons at much higher temperatures. Using this value for $\mathrm{Kr}$ we find a value of the VPIE for $\mathrm{Kr}$ to be $1.98 \times 10^{-4}$ per mass unit at the boiling point. Correcting this to the triple point we obtain the VPIE of $2.33 \times 10^{-4} \pm 0.12 \times 10^{-4}$ while the previously reported value is $2.50 \times 10^{-4}$. The same procedure is then followed for xenon. At the triple point, the VPIE from this work would be $5.28 \times 10^{-5} \pm 2.0 \times 10^{-5}$ while the theoretical value reported by Tew is $5.17 \times 10^{-5}$ per mass unit. Thus, our experimental values and the theoretical values from Tew are consistent.

The slope column gives the slope of the enrichment factor expressed. Vapour Pressure Isotope Effect (VPIE) projected to the triple point are compared with the expectation from the review by Tew. For this review, the values for argon and krypton were experimental values while the xenon value is taken from theory. All VPIE values are changes per unit mass. The value for Ar has been used to normalize our values for krypton and xenon. The agreement between our value for krypton compared with previous work is a measure of the accuracy of the analysis. 
The errors in table (5.6) do not include possible systematic errors due to assuming a value of HETP fixed be the argon data. Our value for krypton agrees well with the earlier data of [1] within the error and this supports the assumption of fixed HETP. For xenon we see good agreement with the theoretical prediction. 


\section{Chapter 6: Conclusion}

The search for evidence of neutrinoless double beta decay is one of the most pressing problems in subatomic physics. To increase the sensitivity of the detectors for such searches, experimenters need ever-larger amounts of the target material. One of the most promising targets is an isotope of xenon, ${ }^{136} \mathrm{Xe}$. This isotope is considered as very important and attracting target for neutrinoless double beta decay, and other studies since it can be made mostly free of the radioactive contamination has excellent self-shielding from external backgrounded can be made into detectors of high sensitivity.

This thesis presents a study of the first step in exploring the feasibility of economical production of this isotope by distillation. Separation by distillation depends on the constituents having different vapour pressures. For xenon, the differences in vapour pressures of the isotopes are expected to be extremely small and direct measurements are very difficult. Therefore, in this thesis, the indirect approach of using distillation itself to measure the effect has been followed.

We have produced the first credible measure of the separation power of a still for xenon isotopes. These lead to the first VPIE data for xenon and these are in good agreement with the theoretical predictions. We also have demonstrated that the predicted linear relationship between VPIE and isotope mass is followed for argon, krypton and xenon. This is the first time this aspect of the theory has been tested. The results will allow a study of the feasibility of distillation as a technique for isotopic enrichment of xenon. The results are also relevant to the use of the triple point of xenon as a temperature standard. 
The VPIE from this work would be $5.28 \times 10^{-5} \pm 2.0 \times 10^{-5}$ while the theoretical value reported by Tew is $5.17 \times 10^{-5}$ per mass unit. Thus, the experimental and theoretical values are consistent with the accuracy of the present slope measurement. For future programming, because the effect is so small (about 1\% change in isotope concentration) a taller column is preferred. When we go to a column 8 times higher, the effect should 8 times larger. Such an experimental column is being installed at SNOLAB. 


\section{Bibliography}

[1] W. L. Tew, "Estimating the Triple-point Isotope Effect and the Corresponding Uncertainties for Cryogenic fixed points," International Journal of

Thermophysics, vol. 29, no. 1, pp. 67-81, 2008.

[2] K. Clusisus, Z. Phys, vol. Abt. B 50, p. 403, 1941.

[3] V. N. Grigor'ev, "THE PRESSURE DIFFERENCE IN SATURATED Kr AND Xe VAPORS," Zhur. Fiz. Khim., vol. 36, 1962.

[4] K. Hill and A. Steele, "The triple point of xenon," Metrologia, vol. 42, no. 4, p. 278, 2005.

[5] W. Furry, "On transition probabilities in double beta-disintegration," Physical Review, vol. 56, no. 12, p. 1184, 1939.

[6] S. Marcocci, F. Vissani, S. Dell'Oro, and M. Viel, "Neutrinoless Double Beta Decay: 2015 Review," Advances in High Energy Physics (Online), vol. 2016, 2016.

[7] J. Albert et al., "Sensitivity and discovery potential of the proposed nEXO experiment to neutrinoless double- $\beta$ decay," Physical Review $C$, vol. 97, no. 6, p. $065503,2018$.

[8] F. Monrabal et al., "The next white (new) detector," Journal of Instrumentation, vol. 13, no. 12, p. P12010, 2018.

[9] A. Gando et al., "Search for Majorana neutrinos near the inverted mass hierarchy region with KamLAND-Zen," Physical review letters, vol. 117, no. 8, p. 082503, 2016.

[10] X. Chen et al., "PandaX-III: Searching for neutrinoless double beta decay with high pressure 136 Xe gas time projection chambers," Science China Physics, Mechanics \& Astronomy, vol. 60, no. 6, p. 061011, 2017.

[11] K. Abe et al., "Atmospheric neutrino oscillation analysis with external constraints in Super-Kamiokande I-IV," Physical Review D, vol. 97, no. 7, p. 072001, 2018.

[12] M. Wascko, "T2k status, results, and plans," in XXVIII International Conference on Neutrino Physics and Astrophysics, 2018.

[13] M. Acero et al., "New constraints on oscillation parameters from $v$ e appearance and $v \mu$ disappearance in the NOvA experiment," Physical Review D, vol. 98, no. 3, p. 032012, 2018.

[14] D. Collaboration., "Design and stats of the new cryogenic distillation column for stable isotope separation,DarkSide collaboration.To be published.," ed.

[15] "When Energy Conservation Seems to Fail: The Prediction of the Neutrino," vol. 23, ed: Springer Nature, 2014, pp. 1339-1359.

[16] R. S. Killick, "Observation of Singly Charged Barium Ions in a Buffer Gas: Towards a Functional Barium-Tagging System for Use in the Enriched Xenon Observatory," Carleton University, 2015. 
[17] C. Ellis and W. Wooster, "The Average Energy of Disintegration of Radium E," Proceedings of the Royal Society of London. Series A, Containing Papers of a Mathematical and Physical Character, vol. 117, no. 776, pp. 109-123, 1927.

[18] E. Fermi, "Versuch einer Theorie der $\beta$-Strahlen. I," Zeitschrift für Physik, journal article vol. 88, no. 3, pp. 161-177, March 01 1934, doi: 10.1007/bf01351864.

[19] F. a. C. J. Reines, CL, "Detection of the free neutrino," in Physical Review vol. 92, ed: APS, 1953, p. 830.

[20] T.-D. Lee and C.-N. Yang, "Question of parity conservation in weak interactions," Physical Review, vol. 104, no. 1, pp. 254-258, 1956.

[21] C.-S. Wu, E. Ambler, R. Hayward, D. Hoppes, and R. P. Hudson, "Experimental test of parity conservation in beta decay," Physical review, vol. 105, no. 4, pp. 1413-1415, 1957.

[22] T. D. Lee and C.-N. Yang, "Parity nonconservation and a two-component theory of the neutrino," Physical Review, vol. 105, no. 5, p. 1671, 1957.

[23] M. Goldhaber, L. Grodzins, and A. Sunyar, "Helicity of neutrinos," Physical Review, vol. 109, no. 3, pp. 1015 - 1017, 1958.

[24] G. Danby et al., "Observation of high-energy neutrino reactions and the existence of two kinds of neutrinos," Physical Review Letters, vol. 9, no. 1, pp. 36-44, 1962.

[25] M. L. Perl et al., "Evidence for anomalous lepton production in e+- eannihilation," Physical Review Letters, vol. 35, no. 22, pp. 1489-1492, 1975.

[26] T. S. Electroweak et al., "Precision electroweak measurements on the Z resonance," Physics Reports, vol. 427, no. 5-6, pp. 257-454, 2006.

[27] K. Kodama et al., "Observation of tau neutrino interactions," Physics Letters B, vol. 504, no. 3, pp. 218-224, 2001.

[28] J. Christenson, J. Cronin, V. Fitch, and R. Turlay, "Evidence for the 2pi Decay of the K20 Meson," in Quasars and high-energy astronomy, Proceedings of the 2nd Texas Symposium on Relativistic Astrophysics, held in Austin, December 15-19, 1964 Edited by KN Douglas, I. Robinson, A. Schild, EL Schucking, JA Wheeler, and NJ Woolf. New York: Gordon \&Breach, 1969, p. 367, 1969, p. 367.

[29] Y. Chao et al., "Evidence for Direct C P Violation in B $0 \rightarrow \mathrm{K}+\pi-$ Decays," Physical review letters, vol. 93, no. 19, p. 191802, 2004.

[30] B. Aubert et al., "Direct C P Violating Asymmetry in B $0 \rightarrow \mathrm{K}+\pi-$ Decays," Physical review letters, vol. 93, no. 13, p. 131801, 2004.

[31] M. P. Rozo Martinez, "Ion Extraction and Counting System for Barium Tagging in Gaseous Xenon for the Enriched Xenon Observatory," Carleton University, 2014.

[32] J. N. Bahcall, "Solar neutrinos. i. theoretical," Physical Review Letters, vol. 12, no. 11, p. 300, 1964.

[33] J. N. Bahcall, N. A. Bahcall, and G. Shaviv, "Present Status of the Theoretical Predictions for the Cl 37 Solar-Neutrino Experiment," Physical Review Letters, vol. 20, no. 21, p. 1209, 1968.

[34] R. Davis Jr, D. S. Harmer, and K. C. Hoffman, "Search for neutrinos from the sun," Physical Review Letters, vol. 20, no. 21, p. 1205, 1968.

[35] B. Pontecorvo, "Neutrino experiments and the problem of conservation of leptonic charge," Sov. Phys. JETP, vol. 26, no. 984-988, p. 165, 1968. 
[36] M. Gell-Mann and A. Pais, "Behavior of neutral particles under charge conjugation," in Murray Gell-Mann: Selected Papers: World Scientific, 2010, pp. 65-67.

[37] K. Lande, E. T. Booth, J. Impeduglia, L. M. Lederman, and W. Chinowsky, "Observation of long-lived neutral V particles," Physical Review, vol. 103, no. 6, p. 1901, 1956.

[38] A. McDonald et al., "Direct Evidence for Neutrino Flavor Transformation from Neutral-Current Interactions in SNO," in AIP Conference Proceedings, 2002, vol. 646, no. 1: AIP, pp. 43-58.

[39] Z. Maki, M. Nakagawa, and S. Sakata, "Remarks on the unified model of elementary particles," Progress of Theoretical Physics, vol. 28, no. 5, pp. 870$880,1962$.

[40] K. Zuber, Neutrino physics. CRC press, 2011.

[41] Review of Particle physics, Particle Data Group (Phys. Rev. D 98, no. 030001). 2018.

[42] S. b. Delaquis, "Construction and operation of the EXO-100 cryogenic facility for $\mathrm{R} \backslash \& \mathrm{D}$ in liquid xenon: advances in barium ion tagging," der Universität Bern, 2015.

[43] P. Minkowski, " $\mu \rightarrow$ e $\gamma$ at a rate of one out of 109 muon decays?," Physics Letters $B$, vol. 67, no. 4, pp. 421--428, 1977.

[44] G. G. Ross, Grand Unified Theories. Westview Press, 1984.

[45] V. Aseev et al., "Upper limit on the electron antineutrino mass from the Troitsk experiment," Physical Review D, vol. 84, no. 11, p. 112003, 2011.

[46] K. Assamagan et al., "Upper limit of the muon-neutrino mass and charged-pion mass from momentum analysis of a surface muon beam," Physical Review D, vol. 53, no. 11, p. 6065, 1996.

[47] R. Barate and A. Collaboration, "An upper limit on the $\$ \backslash$ tau $\$$ neutrino mass from three-and five-prong tau decays," The European Physical Journal C-Particles and Fields, vol. 2, no. 3, pp. 395-406, 1998.

[48] F. Boehm and P. Vogel, Physics of massive neutrinos. Cambridge University Press, 1992.

[49] F. T. Avignone III, S. R. Elliott, and J. Engel, "Double beta decay, Majorana neutrinos, and neutrino mass," Reviews of Modern Physics, vol. 80, no. 2, p. 481, 2008 .

[50] S. Bilenky and C. Giunti, "Neutrinoless double-beta decay: A brief review," Modern Physics Letters A, vol. 27, no. 13, p. 1230015, 2012.

[51] M. J. Dolinski, A. W. Poon, and W. Rodejohann, "Neutrinoless double-beta decay: status and prospects," Annual Review of Nuclear and Particle Science, vol. 69, 2019.

[52] M. Green et al., "Observation of single collisionally cooled trapped ions in a buffer gas," Physical Review A, vol. 76, no. 2, p. 023404, 2007.

[53] C. Chambers et al., "Imaging individual barium atoms in solid xenon for barium tagging in nEXO," arXiv preprint arXiv:1806.10694, 2018.

[54] A. McDonald et al., "Demonstration of single-barium-ion sensitivity for neutrinoless double-beta decay using single-molecule fluorescence imaging," Physical review letters, vol. 120, no. 13, p. 132504, 2018. 
[55] S. Umehara et al., "Neutrino-less double- $\beta$ decay of $\mathrm{Ca} 48$ studied by $\mathrm{CaF} 2$ (Eu) scintillators," Physical Review C, vol. 78, no. 5, p. 058501, 2008.

[56] M. Agostini et al., "Improved Limit on Neutrinoless Double- $\beta$ Decay of Ge 76 from GERDA Phase II," Physical review letters, vol. 120, no. 13, p. 132503, 2018.

[57] C. Aalseth et al., "Search for Neutrinoless Double- $\beta$ Decay in Ge 76 with the Majorana Demonstrator," Physical review letters, vol. 120, no. 13, p. 132502, 2018

[58] A. Barabash, V. Brudanin, and N. Collaboration, "Investigation of double-beta decay with the NEMO-3 detector," Physics of Atomic Nuclei, vol. 74, no. 2, pp. 312-317, 2011.

[59] J. Argyriades et al., "Measurement of the two neutrino double beta decay half-life of Zr-96 with the NEMO-3 detector," Nuclear Physics A, vol. 847, no. 3-4, pp. 168-179, 2010.

[60] R. Arnold et al., "Results of the search for neutrinoless double- $\beta$ decay in Mo 100 with the NEMO-3 experiment," Physical Review D, vol. 92, no. 7, p. 072011, 2015.

[61] R. Arnold et al., "Measurement of the $2 v \beta \beta$ decay half-life and search for the 0 $v \beta \beta$ decay of Cd 116 with the NEMO-3 detector," Physical Review D, vol. 95, no. 1, p. 012007, 2017.

[62] C. Arnaboldi et al., "A calorimetric search on double beta decay of 130Te," Physics Letters B, vol. 557, no. 3-4, pp. 167-175, 2003.

[63] C. Alduino et al., "First Results from CUORE: A Search for Lepton Number Violation via $0 \vee \beta \beta$ Decay of Te 130," Physical review letters, vol. 120, no. 13, p. 132501, 2018.

[64] A. Gando et al., "Publisher's Note: Search for Majorana Neutrinos Near the Inverted Mass Hierarchy Region with KamLAND-Zen [Phys. Rev. Lett. 117, 082503 (2016)]," Physical review letters, vol. 117, no. 10, p. 109903, 2016.

[65] J. Albert et al., "Search for neutrinoless double-beta decay with the upgraded EXO-200 detector," Physical review letters, vol. 120, no. 7, p. 072701, 2018.

[66] R. Arnold et al., "Measurement of the $2 v \beta \beta$ decay half-life of Nd 150 and a search for $0 \vee \beta \beta$ decay processes with the full exposure from the NEMO-3 detector," Physical Review D, vol. 94, no. 7, p. 072003, 2016.

[67] R. Luescher, et al., " Search for $\beta \beta$ decay in 136Xe: new results from the Gotthard experiment.," Physics Letters B, vol. 434, no. 3-4, pp. 407--414, 1998 .

[68] M. Auger et al., "The EXO-200 detector, part I: detector design and construction," Journal of Instrumentation, vol. 7, no. 05, p. P05010, 2012.

[69] N. Ackerman et al., "Observation of Two-Neutrino Double-Beta Decay in Xe 136 with the EXO-200 Detector," Physical Review Letters, vol. 107, no. 21, p. 212501, 2011.

[70] S. A. Kharusi et al., "nEXO pre-conceptual design report," arXiv preprint arXiv:1805.11142, 2018.

[71] H. Qiao, C. Lu, X. Chen, K. Han, X. Ji, and S. Wang, "Signal-background discrimination with convolutional neural networks in the PandaX-III experiment using MC simulation," SCIENCE CHINA Physics, Mechanics \& Astronomy, vol. 61, no. 10, p. 101007, 2018. 
[72] H. C. Urey, "The thermodynamic properties of isotopic substances," Journal of the Chemical Society, pp. 562-81, 1947.

[73] D. C. Dumitrache, B. De Schutter, A. Huesman, and E. Dulf, "Modeling, analysis, and simulation of a cryogenic distillation process for $13 \mathrm{C}$ isotope separation," Journal of Process Control, vol. 22, no. 4, pp. 798-808, 2012.

[74] H. London, "Separation of Isotopes," George Newnes Ld, vol. 66, no. 3, pp. 279279, 1961.

[75] C. J. King, Separation Processes, second ed. New York: McGraw-Hill, 1980.

[76] P. C. Wankat, Separation process engineering, second ed. Upper Saddle River: Prentice Hall, 2006.

[77] J. N. Canongia Lopes, A. A H Pá, L. P N Rebelo, and J. Bigeleisen, Calculation of vapor pressure isotope effects in the rare gases and their mixtures using an integral equation theory. The Journal of Chemical Physics, 2003, pp. 5028-5037.

[78] A. Chialvo and J. Horita, Isotopic effect on phase equilibria of atomic fluids and their mixtures: A direct comparison between molecular simulation and experiment. The Journal of Chemical Physics, 2003.

[79] I. Alekseev et al., "Cryogenic distillation facility for isotopic purification of protium and deuterium," Review of Scientific Instruments, vol. 86, no. 12, p. 125102, 2015.

[80] M. Fenske, "Fractionation of straight-run Pennsylvania gasoline," Industrial \& Engineering Chemistry, vol. 24, no. 5, pp. 482-485, 1932.

[81] B. Death, "Argon, Krypton \& Xenon Isotope Separation by Distillation," p. 8, 2019.

[82] C. I. Company. "PRO-PAK Protruded Metal Distillation Packing, ." https://www.cannoninstrument.com/en/Image/GetDocument/425 (accessed April 20,2019.

[83] G. Santoiemma, "Recent methodologies for studying the soil organic matter," vol. 123, ed. Applied soil ecology: Elsevier, 2018, pp. 546--550.

[84] D. J. Douglas, A. J. Frank, and D. Mao, "Linear ion traps in mass spectrometry," Mass spectrometry reviews, vol. 24, no. 1, pp. 1-29, 2005.

[85] R. J. Hughes and J. F. Todd, Quadrupole storage mass spectrometry. New York; Toronto: Wiley, 1989.

[86] N. McLachan, "Theory and Application of Mathieu Functions, Clarendon," ed: Oxford, 1947.

[87] J. H. Batey, "The physics and technology of quadrupole mass spectrometers," Vacuum, Article vol. 101, pp. 410-415, 03/01/March 2014 2014, doi: 10.1016/j.vacuum.2013.05.005.

[88] I. Wagner, J. Stichlmair, and J. R. Fair, "Mass transfer in beds of modern, highefficiency random packings," Industrial \& engineering chemistry research, vol. 36, no. 1, pp. 227-237, 1997. 